\title{
Smoothing of Limit Linear Series on Curves and Metrized Complexes of Pseudocompact Type
}

\author{
Xiang He
}

\begin{abstract}
We investigate the connection between Osserman limit series (on curves of pseudocompact type) and Amini-Baker limit linear series (on metrized complexes with corresponding underlying curve) via a notion of pre-limit linear series on curves of the same type. Then, applying the smoothing theorems of Osserman limit linear series, we deduce that, fixing certain metrized complexes, or for certain types of Amini-Baker limit linear series, the smoothability is equivalent to a certain "weak glueing condition". Also for arbitrary metrized complexes of pseudocompact type the weak glueing condition (when it applies) is necessary for smoothability. As an application we confirm the lifting property of specific divisors on the metric graph associated with a certain regular smoothing family, and give a new proof of a result of Cartright, Jensen, and Payne for vertex-avoiding divisors, and generalize it for divisors of rank one in the sense that, for the metric graph, there could be at most three edges (instead of two) between any pair of adjacent vertices.
\end{abstract}

\section{Introduction}

The theory of limit linear series has been developed by Eisenbud and Harris in [EH86] for handling the degeneration of linear series on smooth curves as the curves degenerate to reducible curves (of compact type). It has been applied to prove results involving moduli space of curves, such as the Brill-Noether theorem ([GH80]), the GiesekerPetri theorem ([Gie82]), and that moduli spaces of curves of sufficiently high genus are of general type ([HM82, EH87]), etc.

A complete generalization of Eisenbud-Harris theory has remained open. Earlier approaches can be found in papers of E. Esteves such as [Est98, EM02]. Recently, Amini and Baker [AB15] introduced a notion of metrized complexes, which is roughly speaking a finite metric graph $\Gamma$ together with a collection of marked curves $C_{v}$, one for each vertex $v$, such that the marked points of $C_{v}$ is in bijection with the edges of $\Gamma$ incident to $v$. This can be considered as an enrichment of both metric graphs and nodal curves. The concept of limit linear series on a metrized complex is proposed in [AB15] as well as the specialization map. While the Amini-Baker limit linear series satisfies the specialization theorem, it is unclear how to prove a general theorem about

Received by the editors July 26, 2017; revised June 23, 2018.

Published online on Cambridge Core M arch 15, 2019.

AMS subject classification: $14 \mathrm{H} 60,14 \mathrm{~T} 05$.

Keywords: curves, limit linear series, metrized complex. 
their smoothing behaviors. Related results can be found in [LM14], where a sufficient and necessary condition for the smoothability (with respect to certain base field) of a (saturated) limit linear series of rank one is given.

On the other hand, B. Osserman [Oss] developed a notion of limit linear series on curves possibly not of compact type, which is a generalization of Eisenbud and Harris limit linear series. He also proved the specialization theorem, as well as a smoothing theorem for curves of pseudocompact type (see below for definition), which states that a limit linear series is smoothable if the moduli space is of expected dimension at the corresponding point. This improves the smoothing theorem in the compact-type case in the sense that it applies for possibly non-refined limit linear series.

In this paper we investigate the smoothing of Amini-Baker limit linear series by studying their connection with Osserman limit linear series.

Let $X_{0}$ be a curve of pseudocompact type, which is a curve whose dual graph $G$ is a multigraph obtained from a tree $\bar{G}$ by adding parallel edges between adjacent vertices of $\bar{G}$. A chain structure $n$ on $G$ is roughly an integer-valued length function on $E(G)$. This induces a metric graph $\Gamma$ as well as a metrized complex $\mathfrak{C}_{X_{0}, n}$ with underlying graph $\Gamma$ (see $\$ 2$ for details). Given a chain structure $n$, let $\widetilde{X}_{0}$ be the curve obtained from $X_{0}$ by inserting a chain of $n(e)-1$ projective lines at the node of $e$ for all $e \in E(G)$. Let $\widetilde{G}$ be the dual graph of $\widetilde{X}_{0}$. An Osserman limit linear series (which we also refer to as a limit linear series on $\left(X_{0}, n\right)$ ) then consists of a certain line bundle $\mathscr{L}$ on $\widetilde{X}_{0}$ and a collection of linear systems on each component of $X_{0}$ that satisfies certain (multi)vanishing conditions. An Amini-Baker limit linear series (which we also refer to as a limit linear series on $\left.\mathfrak{C}_{X_{0}, n}\right)$ consists of a divisor on $\mathfrak{C}_{X_{0}, n}$ with a collection of linear spaces of rational functions on each component of $X_{0}$ satisfying certain rank conditions. The multidegree $w_{0}$ of a limit linear series on $\left(X_{0}, n\right)$ is the multidegree of $\mathscr{L}$ on $V(\widetilde{G})$, which induces a divisor on $\Gamma$ as we identify $\Gamma$ with the metric graph obtained from $\widetilde{G}$ by assigning length 1 to every edge. We also say a limit linear series on $\mathfrak{C}_{X_{0}, n}$ of multidegree $w_{0}$ if its underlying divisor on $\Gamma$ is (up to linear equivalence) induced by $w_{0}$.

In the following we fix $w_{0}$ to be the multidegree of a limit linear series on $\left(X_{0}, \boldsymbol{n}\right)$ and $\mathfrak{C}_{X_{0}, n}$. Note that, by the definition of Osserman limit linear series, $w_{0}$ is assumed to be "admissible", namely, when restricting to each component of $\Gamma \backslash V(G)$, the corresponding divisor $D$ of $w_{0}$ is effective and of at most degree one; see also Definition 2.1.

In [Oss17] Osserman constructed a map $\mathfrak{F}$ from the set of limit linear series on $\left(X_{0}, n\right)$ to the set of limit linear series on $\mathfrak{C}_{X_{0}, n}$. It is also proved in [Oss17] that $\mathfrak{F}$ can be defined over the set of pre-limit linear series (a weakened version of Osserman limit linear series); in this case we show in Section 3 that $\mathfrak{F}$ is a bijection. This essentially says that a limit linear series on $\mathfrak{C}_{X_{0}, n}$ carries the same data as a pre-limit linear series on $\left(X_{0}, n\right)$. We also give a necessary condition for a pre-limit linear series (hence for a limit linear series on $\left.\mathfrak{C}_{X_{0}, n}\right)$ to be lifted to a limit linear series on $\left(X_{0}, n\right)$, called the weak glueing condition.

In Section 4 we consider the smoothing of limit linear series on $\mathfrak{C}_{X_{0}, n}$. We show in Theorem 4.7 that a necessary condition is the weak glueing condition. On the other hand, it is proved in [Oss16] that for a special case of $\left(X_{0}, n\right)$ the moduli space of limit linear series (again of multidegree $w_{0}$ ) is of expected dimension, hence every limit 
linear series on $\left(X_{0}, n\right)$ is smoothable. We show in this case that the weak glueing condition is sufficient for a pre-limit linear series to be lifted to a limit linear series, and use the "equivalence" between pre-limit linear series on $\left(X_{0}, n\right)$ and limit linear series on $\mathfrak{C}_{X_{0}, n}$ to give a smoothing theorem for the latter (see also Theorem 4.8).

Theorem 1.1 Suppose $\left(X_{0}, n\right)$ satisfies the conditions of Theorem 4.3. In other words, the induced metric graph $\Gamma$ has few edges, the edge lengths of $\Gamma$ are general with respect to a fixed degree $d^{\prime}$, and the components of $X_{0}$ are strongly Brill-Noether general. Then a limit linear series on $\mathfrak{C}_{X_{0}, n}$ of degree not exceeding $d^{\prime}$ is smoothable if and only if it satisfies the weak glueing condition.

Additionally, for arbitrary $n$ and $X_{0}$ with strongly Brill-Noether general components we consider a family of $w_{0}$ such that the induced divisor $D$ on $\Gamma$ is "randomly distributed" on $\Gamma \backslash V(G)$, as in Theorem 4.4. More precisely, for any edge $e \in E(\bar{G})$, let $e_{1}^{\circ}, \ldots, e_{m}^{\circ}$ be the components of $\Gamma \backslash V(G)$ corresponding to edges $e_{1}, \ldots, e_{m} \in E(G)$ that lies over $e$. Given a certain direction on $G$, the divisor $\left.D\right|_{e_{i}^{\circ}}$ gives an integer $x_{i}$ in $\left[0, n\left(e_{i}\right)-1\right]$, and we consider $w_{0}$ such that $x_{1}, \ldots, x_{m}$ are distinct modulo $\operatorname{gcd}\left(\boldsymbol{n}\left(e_{1}\right), \ldots, \boldsymbol{n}\left(e_{m}\right)\right)$. We show that a pre-limit linear series automatically lifts to a limit linear series on $\left(X_{0}, n\right)$, and the dimension of the moduli space of limit linear series on $\left(X_{0}, n\right)$ is as expected. In this case, any limit linear series on $\mathfrak{C}_{X_{0}, n}$ is smoothable.

In Section 5 we consider the problem of lifting divisors on the metric graph $\Gamma$ to the generic fiber $X_{\eta}$ of any regular smoothing family $X$ (Definition 2.11) with special fiber $\widetilde{X}_{0}$ with rational components and dual graph $\widetilde{G}$. When $\Gamma$ is a generic chain of loops, it is proved by reducing to the so-called vertex-avoiding divisors that every rational divisor on $\Gamma$ is liftable (see [CJP15] for details). For $\left(X_{0}, n\right)$ as in Theorem 1.1, again since the weak glueing condition is sufficient for a pre-limit linear series to be lifted to a limit linear series on $\left(X_{0}, n\right)$, and the dimension counting shows that every limit linear series on $\left(X_{0}, n\right)$ is smoothable, we are able to prove the following theorem, which gives an alternative approach to lifting (rational) vertex-avoiding divisors on a generic chain of loops, by lifting the divisor on $\Gamma$ to a pre-limit linear series on $\left(X_{0}, n\right)$ that satisfies the weak glueing condition.

Theorem 1.2 Let $\left(X_{0}, n\right)$ be as in Theorem 1.1 and let $X$ be as above. Suppose further that $X_{0}$ only has rational components, and that $\bar{G}$ is a path graph. Then every rational divisor on $\Gamma$ of rank less than or equal to 1 lifts to a divisor on $X_{\eta}$ of the same rank. In addition, if $\Gamma$ is a generic chain of loops, then every rational vertex-avoiding divisor lifts to a divisor on $X_{\eta}$ with the same rank.

See Theorems 5.1 and 5.4 for more precise statements. Note that, ignoring the field condition (see below), the first part of the theorem confirms [CDPR12, Conjecture 1.5] and [CJP15, Theorem 1.1] for divisors of rank one, and generalizes the conditions in [CJP15, Theorem 1.1] for the graph $\Gamma$, in the sense that there could be at most three edges (instead of two) between any pair of adjacent vertices. 


\subsection{Conventions and Notation}

All curves we consider are proper, (geometrically) reduced and connected, and at worst nodal. All nodal curves are split. All irreducible components of a nodal curve are smooth.

In the sequel we let $R$ be a complete discrete valuation ring with valuation val: $R \rightarrow$ $\mathbb{Z}_{>0}$ and residue field $\kappa$. Let $K$ be the fraction field of $R$, which is a non-archimedean field with the induced norm $|x|=\exp (-\operatorname{val}(x))$. Let $\widetilde{K}$ be the completion of the algebraic closure of $K$ and $\widetilde{R}$ the valuation ring of $\widetilde{K}$. Note that $\widetilde{K}$ is still algebraically closed, the valuation/norm extends uniquely to $\widetilde{K}$, and $\widetilde{K}$ has residue field $\kappa$ if $\kappa$ is algebraically closed ( $c f$. [Con $08, \$ 1.1])$.

Let $X_{0}$ be a curve with dual graph $G$. For $v \in V(G)$ let $Z_{v}$ be the irreducible component of $X_{0}$ corresponding to $v$. For $e^{\prime} \in E(G)$ that is incident to $v$ let $P_{e^{\prime}}$ be the node of $X_{0}$ corresponding to $e^{\prime}$ and $P_{e^{\prime}}^{v}$ the preimage (of the normalization map of $X_{0}$ ) of $P_{e^{\prime}}$ in $Z_{v}$. Let $\bar{G}$ be the graph obtained from $G$ as follows: for each pair of adjacent vertices $v$ and $v^{\prime}$, replace all edges connecting $v$ and $v^{\prime}$ by a single edge. For $e \in E(\bar{G})$, we denote

$$
\mathcal{A}_{e}^{v}=\bigcup_{e^{\prime}} P_{e^{\prime}}^{v} \quad \text { and } \quad \mathcal{A}_{v}=\bigcup_{e \ni v} \mathcal{A}_{e}^{v},
$$

where in the first expression $e^{\prime}$ runs through all edges of $G$ that lies over $e$.

For a curve (graph, metric graph, metrized complex) $\mathscr{X}$ denote by $\operatorname{Div}(\mathscr{X})$ the space of divisors on $\mathscr{X}$. If $\mathscr{X}$ is a curve let $K(\mathscr{X})$ be the space of rational functions on $\mathscr{X}$; if $\mathscr{X}$ is a graph (metric graph, metrized complex), denote the space of rational functions on $\mathscr{X}$ by $\operatorname{Rat}(\mathscr{X})$. Given a rational function $f$ on $\mathscr{X}$, we denote by $\operatorname{div}(f)$ the associated divisor.

Let $C$ be a curve. Let $\mathscr{O}_{C}(D)$ be an invertible sheaf on $C$ where $D \in \operatorname{Div}(C)$ and take a nonzero section $s \in H^{0}\left(C, \mathscr{O}_{C}(D)\right)$. We denote by $\operatorname{div}^{0}(s)$ the effective divisor associated with $s$ that is rationally equivalent to $D$. For a divisor $D^{\prime} \in \operatorname{Div}(C)$ and $P \in C$ denote by $\operatorname{ord}_{P}\left(D^{\prime}\right)$ the coefficient of $P$ in $D^{\prime}$, denote $\operatorname{ord}_{P}^{0}(s)=\operatorname{ord}_{P}\left(\operatorname{div}^{0}(s)\right)$. For a rational function $f \in K(C)$, let $\operatorname{ord}_{P}(f)$ be the vanishing order of $f$ at $P$. Hence, if $s$ is considered as a rational function, then we have $\operatorname{ord}_{P}^{0}(s)=\operatorname{ord}_{P}(s)+\operatorname{ord}_{P}(D)$.

Let $\Gamma$ be a metric graph with underlying graph $G$. For $e \in E(G)$ and $v \in V(G)$ incident on $e$ and $f \in \operatorname{Rat}(\Gamma)$, let $\operatorname{slp}_{e, v}(f)$ be the outgoing slope of $f$ at $v$ along the tangent direction corresponding to $e$. For $x \in \Gamma$, let $\operatorname{ord}_{x}(f)$ be the sum of outgoing slopes of $f$ over all tangent directions of $x$.

\section{Preliminaries}

We recall some relative notions about Osserman and Amini-Baker limit linear series. Let $G$ be a graph without loops. Recall that a chain structure on $G$ is a function $n: E(G) \rightarrow \mathbb{Z}_{>0}$. Let $\Gamma$ be the corresponding metric graph with edge length defined by $n$, and let $\widetilde{G}$ be the graph obtained from $G$ by inserting $n(e)-1$ vertices between the vertices adjacent to $e$ for all $e \in E(G)$. Then $\Gamma$ can also be obtained from $\widetilde{G}$ by associating unit edge lengths to all of $E(\widetilde{G})$. We use $V(G)$ or $V(\widetilde{G})$ instead of $V(\Gamma)$ for different choices of vertex sets of $\Gamma$. 


\subsection{Some Notions for Graph Theory}

We recall some concepts about admissible multidegrees ([Oss16]) on a graph as well as reduced divisors ([Luol1]).

Definition 2.1 An admissible multidegree $w=\left(w_{G}, \mu\right)$ of total degree $d$ on $(G, n)$ consists of a function $w_{G}: V(G) \rightarrow \mathbb{Z}$ together with a tuple $(\mu(e))_{e \in E(G)} \in \Pi_{e \in E(G)} \mathbb{Z} / n(e) \mathbb{Z}$ such that $d=\#\{e \in E(G) \mid \mu(e) \neq 0\}+\sum_{v \in V(G)} w_{G}(v)$.

Correspondingly, we define the notion of (integral) edge-reduced divisor, which is closely related to admissible multidegree, as follows.

Definition 2.2 A point $x$ on $\Gamma$ is called a rational point (resp., integral point) if the distance between $x$ and any vertex of $\Gamma$ is a rational number (resp., integer).

Definition 2.3 A divisor $D$ on $\Gamma$ is edge-reduced if the restriction of $D$ on each connected component of $\Gamma \backslash V(G)$ is either empty or an effective divisor of degree one. We say that $D$ is rational (resp., integral) if $D$ is supported on rational (resp., integral) points.

Suppose $G$ is directed. There is a natural bijection $\varphi$ between the set of integral edge-reduced divisors on $\Gamma$ (of degree $d$ ) and the set of admissible multidegrees on $(G, n)$ (of degree $d$ ), as follows. Let $D$ be an integral and edge-reduced divisor on $\Gamma$. We set $w_{G}(v)=\operatorname{deg}\left(\left.D\right|_{v}\right)$ for all $v \in V(G)$. For all $e \in E(G)$ with tail $v$, denote by $\tilde{e}$ the edge of $\Gamma$ corresponding to $e$. If $\left.D\right|_{\tilde{e}^{\circ}}=\varnothing$, we set $\mu(e)=0$; otherwise, let $\mu(e)$ be the distance between the point in $\left.D\right|_{\tilde{e}^{\circ}}$ and $v$. We set $D_{w}=\varphi^{-1}(w)$ for any admissible multidegree $w$. See Example 2.6 for an example of an admissible multidegree and the corresponding integral edge-reduced divisor.

For each pair of an edge $e$ and an adjacent vertex $v$, let $\sigma(e, v)=1$ if $v$ is the tail of $e$ and -1 otherwise. We have the following definition of twisting.

Definition 2.4 Let $w=\left(w_{G}, \mu\right)$ be an admissible multidegree on $(G, n)$ and $v \in$ $V(G)$. For each $e \in E(G)$ incident to $v$ we perform the following operations:

(i) If $\mu(e)+\sigma(e, v)=0$, increase $w_{G}\left(v^{\prime}\right)$ by 1 where $v^{\prime}$ is the other vertex of $e$.

(ii) If $\mu(e)=0$, decrease $w_{G}(v)$ by 1 .

(iii) Increase $\mu(e)$ by $\sigma(e, v)$.

The resulting admissible multidegree is called the twist of $w$ at $v$. The negative twist of $w$ at $v$ is the admissible multidegree $w^{\prime}$ such that the twist of $w^{\prime}$ at $v$ is equal to $w$.

Let $w_{0}$ be an admissible multidegree. We denote by $G\left(w_{0}\right)$ the directed graph with vertex set consisting of all admissible multidegrees obtained from $w_{0}$ by sequences of twists, and with an edge from $w$ to $w^{\prime}$ if $w^{\prime}$ is obtained from $w$ by twisting at some vertex $v \in V(G)$. Given $w \in V\left(G\left(w_{0}\right)\right)$ and $v_{1}, \ldots, v_{m} \in V(G)$ (not necessarily distinct), let $P\left(w, v_{1}, \ldots, v_{m}\right)$ denote the path in $V\left(G\left(w_{0}\right)\right)$ obtained by starting at $w$ and twisting successively at each $v_{i}$. 
Remark 2.5 In the case of an integral edge-reduced divisor $D$ on $\Gamma$ twisting at $v$ (where we choose $V(G)$ as the vertex set of $\Gamma$ ) is the same as performing the following operation on $D$ : for all $e \in E(\Gamma)$ incident to $v$, if $\left.D\right|_{e^{\circ}}$ is nonempty replace the point $\left.p \in D\right|_{e^{\circ}}$ by the unique point on $e$ whose distance to $v$ is one more than the distance between $p$ and $v$; otherwise, decrease $\operatorname{deg}\left(\left.D\right|_{v}\right)$ by one and add to $D$ the unique point on $e$ whose distance to $v$ is one. Hence the definition of twisting is independent of the direction of $G$, and we get a linearly equivalent divisor after twisting.

Example 2.6 Consider a graph $G$ consisting of two vertices $v$ and $v^{\prime}$ connected by three edges $e_{1}, e_{2}$, and $e_{3}$. Take a chain structure $n$ with $n\left(e_{1}\right)=4, n\left(e_{2}\right)=2, n\left(e_{3}\right)=3$ and a direction from $v$ to $v^{\prime}$. Let $w=\left(w_{G}, \mu\right)$ where $w_{G}(v)=3, w_{G}\left(v^{\prime}\right)=0, \mu\left(e_{1}\right)=1$, $\mu\left(e_{2}\right)=1$, and $\mu\left(e_{3}\right)=0$. Then $D_{w}$ is as in the left of the following graphs, with each number representing the coefficient of the corresponding node in $D_{w}$.
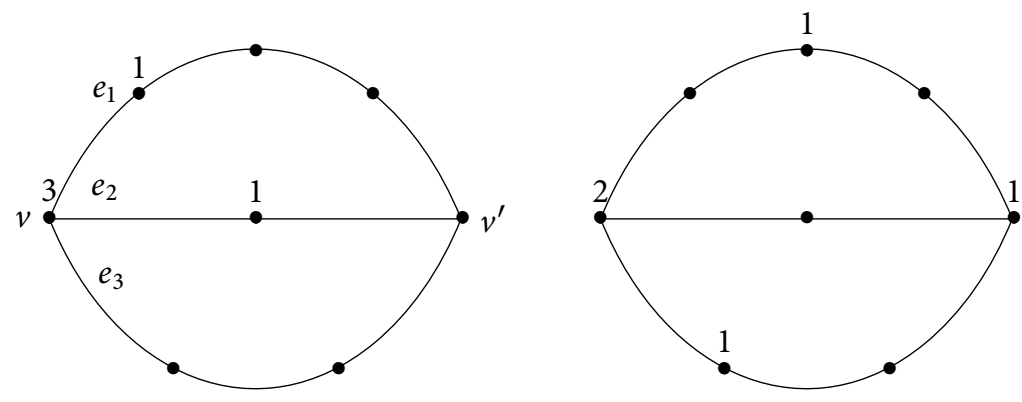

After twisting at $v$, we get $w^{\prime}=\left(w_{G}^{\prime}, \mu^{\prime}\right)$ where $w_{G}^{\prime}(v)=2, w_{G}^{\prime}\left(v^{\prime}\right)=1, \mu^{\prime}\left(e_{1}\right)=2$, $\mu^{\prime}\left(e_{2}\right)=0$ and $\mu^{\prime}\left(e_{3}\right)=1$. The induced $D_{w^{\prime}}$ is given in the right graph.

Let $\bar{G}$ denote the graph obtained from $G$ by replacing parallel edges between any pair of adjacent vertices by a single edge. We say that $G$ is a multitree if $\bar{G}$ is a tree. Any graph with two vertices is a multitree. For multitrees we also consider partial twists.

Definition 2.7 Let $G$ be a multitree, let $(e, v)$ be a pair of an edge $e$ and an incident vertex $v$ of $\bar{G}$, and let $w$ be an admissible multidegree on $(G, n)$. We say that an edge $\tilde{e}$ of $G$ lies over $e$ if the set of vertices of $G$ incident on $\widetilde{e}$ is the same as the set of vertices of $\bar{G}$ incident on $e$. The twist of $w$ at $(e, v)$ is the admissible multidegree obtained from $w$ by performing the operations as in Definition 2.4 only for all edges in $G$ that lies over $e$.

Again for an integral edge-reduced divisor on $\Gamma$, twisting at $(e, v)$ is just performing the operation in Remark 2.5 only for all edges lying over $e$. Note that the twists are commutative, and $w$ remains the same after twisting at all vertices of $G$. Hence the twists are invertible, as the negative twist at $v$ is the same as the composition of the twists at all $v^{\prime} \neq v$. In addition, multitree twisting $w$ at $(e, v)$ is the same as twisting at all vertices $v^{\prime}$ in the connected component of $\bar{G} \backslash\{e\}$ that contains $v$. If $v^{\prime}$ is the other vertex of $e$, then twisting at $(e, v)$ is the inverse of twisting at $\left(e, v^{\prime}\right)$. 
Definition 2.8 Let $G$ be a multitree. An admissible multidegree $w$ is concentrated on $v$ if there is an ordering on $V(G)$ starting at $v$ such that for each subsequent vertex $v^{\prime}$, we have that $w$ becomes negative in vertex $v^{\prime}$ after taking the composition of the negative twists at all previous vertices. A tuple $\left(w_{v}\right)_{v}$ of admissible multidegrees is tight if $w_{v}$ is concentrated at $v$ for all $v \in V(G)$ and for all $e \in E(\bar{G})$ incident to the vertices $v_{1}$ and $v_{2}$, we have that $w_{v_{1}}$ is obtained from $w_{v_{2}}$ by twisting $b_{v_{1}, v_{2}}$ times at $\left(e, v_{2}\right)$ for some $b_{v_{1}, v_{2}} \in \mathbb{Z}_{\geq 0}$.

If $G$ has only two vertices $v$ and $v^{\prime}$, then $w$ being concentrated on $v$ is the same as $w$ being negative at $v^{\prime}$ after twisting at $\left(e, v^{\prime}\right)$. An example of a tight tuple of admissible multidegrees is given by the reduced divisors.

Definition 2.9 Given a vertex $v_{0} \in V(G)$, a divisor $D$ on $G$ is $v_{0}$-reduced if

(i) $D$ is effective on $V(G) \backslash\left\{v_{0}\right\}$;

(ii) for every nonempty subset $S \subset V(G) \backslash\left\{v_{0}\right\}$, there is some $v \in S$ such that $D(v)$ (the coefficient of $v$ in $D$ ) is strictly smaller than the number of edges from $v$ to $V(G) \backslash S$.

Given a point $x_{0} \in \Gamma$ (recall that $\Gamma$ is a metric graph), a divisor $D \in \operatorname{Div}(\Gamma)$ is $x_{0}$-reduced if

(a) $D$ is effective on $\Gamma \backslash\left\{x_{0}\right\}$;

(b) for any closed connected subset $A \subset \Gamma$ there is a point $x \in \partial A$ such that $D(x)$ is strictly less than the number of tangent directions of $\Gamma \backslash(A \backslash x)$ at $x$.

For every divisor $D \in \operatorname{Div}(G)$ (resp., $D \in \operatorname{Div}(\Gamma)$ ) and $v_{0} \in V(G)$ (resp., $x_{0} \epsilon$ $\Gamma$ ), there is a unique divisor $D_{v_{0}}$ (resp., $D_{x_{0}}$ ) such that $D_{v_{0}}$ (resp., $D_{x_{0}}$ ) is linearly equivalent to $D$ and $v_{0}$-reduced (resp., $x_{0}$-reduced). One easily checks that a divisor $D \in \operatorname{Div}(\widetilde{G})$ is $v_{0}$-reduced if and only if it is $v_{0}$-reduced as a divisor on $\Gamma$.

Proposition 2.10 Let $G$ be a multitree. Let $w_{v}^{\text {red }} \in V\left(G\left(w_{0}\right)\right)$ be the admissible multidegree such that $D_{w_{v}^{\text {red }}}$ is $v$-reduced. Then $w_{v}^{\text {red }}$ is the unique admissible multidegree in $V\left(G\left(w_{0}\right)\right)$ that is concentrated on $v$ and nonnegative on all $v^{\prime} \neq v$, and $\left(w_{v}^{\mathrm{red}}\right)_{v}$ is a tight tuple.

Proof The first conclusion follows directly from [Oss17, Corollary 3.9]. According to Dhar's burning algorithm ([Luoll, Algorithm 2.5]), for $v$ and $v^{\prime}$ in $V(\bar{G})$ adjacent to $e \in E(\bar{G})$, we have that $w_{v^{\prime}}^{\text {red }}$ is obtained from $w_{v}^{\text {red }}$ by twisting $b$ times at $(e, v)$, where $b$ is the largest number (possibly negative) such that the resulting multidegree is effective on $V(\bar{G}) \backslash\left\{v^{\prime}\right\}$.

\subsection{Limit Linear Series on Curves of Pseudocompact Type}

In this subsection we recall the definition of limit linear series by Osserman in [Oss16] for curves of pseudocompact type. Note that the notion of limit linear series for more general curves is given in [Oss, Definition 2.21]. 
Definition 2.11 We say that $\pi: X \rightarrow B$ is a smoothing family if $B$ is the spectrum of a DVR, and

(i) $\pi$ is flat and proper;

(ii) the special fiber $X_{0}$ of $\pi$ is a (split) nodal curve;

(iii) the generic fiber $X_{\eta}$ is smooth;

(iv) $\pi$ admits sections through every component of $X_{0}$.

If, further, $X$ is regular, we say that $X$ is a regular smoothing family.

Let $X_{0}$ be a curve over $\kappa$ with dual graph $G$. For $v \in V(G)$, let $Z_{v}$ be the corresponding irreducible component of $X_{0}$ and let $Z_{v}^{c}$ be the closure of the complement of $Z_{v}$.

Definition 2.12 ([Mai98]) An enriched structure on $X_{0}$ consists of, for each $v \in$ $V(G)$ a line bundle $\mathscr{O}_{v}$ on $X_{0}$, the data satisfying

(i) for any $v \in V(G)$, we have $\left.\mathscr{O}_{v}\right|_{Z_{v}} \cong \mathscr{O}_{Z_{v}}\left(-\left(Z_{v}^{c} \cap Z_{v}\right)\right)$ and $\left.\mathscr{O}_{v}\right|_{Z_{v}^{c}} \cong \mathscr{O}_{Z_{v}^{c}}\left(Z_{v}^{c} \cap Z_{v}\right)$;

(ii) $\otimes_{v \in V(G)} \mathscr{O}_{v} \cong \mathscr{O}_{X_{0}}$.

Note that an enriched structure is always induced by any regular smoothing of $X_{0}$ (cf. [Oss, Proposition 3.10]).

Take a chain structure $n$ on $G$ and an admissible multidegree $w_{0}$. Let $\widetilde{X}_{0}$ be the nodal curve obtained from $X_{0}$ by, for each $e \in E(G)$, inserting a chain of $n(e)-1$ projective lines at the corresponding node, and $\widetilde{G}$ the dual graph of $\widetilde{X}_{0}$. We say that a divisor on $\widetilde{G}$ is of multidegree $w_{0}$ if (considered as a divisor on $\Gamma$ ) it is equal to $D_{w_{0}}$, and a line bundle $\mathscr{L}$ on $\widetilde{X}_{0}$ is of multidegree $w_{0}$ if its associated divisor on $\widetilde{G}$ is.

Given an enriched structure $\left(\mathscr{O}_{v}\right)_{v \in V(\widetilde{G})}$ on $\widetilde{X}_{0}$ and a tuple of admissible multidegrees $\left(w_{v}\right)_{v \in V(G)}$ in $V\left(G\left(w_{0}\right)\right)$ such that $w_{v}$ is concentrated on $v$ and a line bundle $\mathscr{L}$ on $\widetilde{X}_{0}$ of multidegree $w_{0}$, we get a tuple of line bundles $\left(\mathscr{L}_{w_{v}}\right)_{v \in V(G)}$. Roughly speaking, suppose $w_{v}$, as a divisor on $\widetilde{G}$, is obtained from $w_{0}$ by firing chips ( $c f$. [BS13, $\left.\$ 4\right]$ ) at a sequence $S \subset V(\widetilde{G})$ of vertices; then $\mathscr{L}_{w_{v}}$ is obtained from $\mathscr{L}$ by tensoring with $\mathscr{O}_{v}$ for all $v$ in $S$. Note that $\mathscr{L}_{w_{v}}$ is of multidegree $w_{v}$. See [Oss16, \$2] for details of this construction.

Definition 2.13 A curve over $\kappa$ is of pseudocompact type if its dual graph is a multitree.

Now suppose $X_{0}$ is a curve of pseudocompact type and the tuple $\left(w_{v}\right)_{v}$ is tight. For each pair $(e, v)$ consisting of an edge $e$ and an incident vertex $v$ of $\bar{G}$, let $\left(D_{i}^{e, v}\right)_{i \geq 0}$ be the effective divisors on $Z_{v}$ defined by setting $D_{0}^{e, v}=0$ and

$$
D_{i+1}^{e, v}-D_{i}^{e, v}=\sum_{\substack{e^{\prime} \in E(G), e^{e^{\prime}} \text { lies over } e \\ \sigma\left(e^{\prime}, v\right) \mu_{v}\left(e^{\prime}\right) \equiv-i}} P_{e^{\prime}}^{v},
$$

where $\mu_{v}: E(G) \rightarrow \mathbb{Z} / \boldsymbol{n}(e) \mathbb{Z}$ is induced by $w_{v}$. Intuitively, $D_{i}^{e, v}$ records the chips we lose (in every direction) at vertex $v$ when twisting $i$ times at $(e, v)$ from $w_{v}$. 
Example 2.14 In Example 2.6, let $w_{v}=w$ and let $w_{v^{\prime}}$ be obtained from $w_{v}$ by twisting three times at $(e, v)$. It is easy to check that $\left(w_{v}, w_{v^{\prime}}\right)$ is a tight tuple. Straightforward calculation shows that

$$
D_{i}^{e, v}=0, P_{e_{3}}^{v}, P_{e_{2}}^{v}+P_{e_{3}}^{v}, P_{e_{2}}^{v}+P_{e_{3}}^{v}, P_{e_{1}}^{v}+2 P_{e_{2}}^{v}+2 P_{e_{3}}^{v}, \ldots
$$

for $i=0,1,2,3,4, \ldots$.

For convenience we call $D_{i}^{e, v}$ the twisting divisors associated with $\left(w_{v}\right)_{v}$. In order to define limit linear series, we have the following glueing isomorphism.

Proposition 2.15 ([Oss16, Proposition 2.14]) Let $X_{0}$ be a curve of pseudocompact type and let $\widetilde{X}_{0},\left(w_{v}\right)_{v}, \mathscr{L}, \mathscr{L}_{w_{v}}$ be as above. Denote $\mathscr{L}^{v}=\left.\mathscr{L}_{w_{v}}\right|_{Z_{v}}$. Take vertices $v$ and $v^{\prime}$ of $\bar{G}$ connected by an edge $e$. Then for $0 \leq i \leq b_{v, v^{\prime}}$, we have isomorphisms

$$
\varphi_{i}^{e, v}: \mathscr{L}^{v}\left(-D_{i}^{e, v}\right) / \mathscr{L}^{v}\left(-D_{i+1}^{e, v}\right) \rightarrow \mathscr{L}^{v^{\prime}}\left(-D_{b_{v, v^{\prime}}}^{e, v^{\prime}}\right) / \mathscr{L}^{v^{\prime}}\left(-D_{b_{v, v^{\prime}}+1-i}^{e, v^{\prime}}\right)
$$

Note that in the above proposition, if $D_{i+1}^{e, v}-D_{i}^{e, v}=\sum_{e^{\prime} \in I} P_{e^{\prime}}^{v}$, then

$$
D_{b_{v, v^{\prime}}}^{e, v^{\prime}}-D_{b_{v, v^{\prime}}}^{e, v^{\prime}}=\sum_{e^{\prime} \in I} P_{e^{\prime}}^{v^{\prime}}
$$

In particular, we have $\operatorname{deg}\left(D_{i+1}^{e, v}-D_{i}^{e, v}\right)=\operatorname{deg}\left(D_{b_{v, v^{\prime}}-i+1}^{e, v^{\prime}}-D_{b_{v, v^{\prime}}-i}^{e, v^{\prime}}\right)$ for all $i$.

Definition $2.16 \quad$ (i) Let $Z$ be a smooth curve and $r, d \geq 0$. Let $D_{0} \leq \cdots \leq D_{b+1}$ be a sequence of effective divisors on $X$. We say $j$ is critical for $D_{\bullet}$ if $D_{j+1} \neq D_{j}$.

(ii) Suppose further that $D_{0}=0$ and $\operatorname{deg} D_{b+1}>d$. Given $(\mathscr{L}, V)$ a $\mathfrak{g}_{d}^{r}$ on $Z$, we define the multivanishing sequence of $(\mathscr{L}, V)$ along $D_{\bullet}$ to be the sequence

$$
a_{0} \leq \cdots \leq a_{r},
$$

where a value $a$ appears in the sequence $m$ times if for some $i$ we have $\operatorname{deg} D_{i}=a$ and $\operatorname{deg} D_{i+1}>a$, and $\operatorname{dim}\left(V\left(-D_{i}\right) / V\left(-D_{i+1}\right)\right)=m$.

(iii) Given $s \in V$ nonzero, the order of vanishing $\operatorname{ord}_{D_{\bullet}}(s)$ along $D_{\bullet}$ is $\operatorname{deg} D_{i}$ where $i$ is maximal so that $s \in V\left(-D_{i}\right)$.

One checks easily that $j$ is critical for $D_{\bullet}^{e, v}$ if and only if $b_{v, v^{\prime}}-j$ is critical for $D_{\bullet}^{e, v^{\prime}}$. We are now able to state the definition of limit linear series on a curve of pseudocompact type (cf. [Oss16, Definition 2.16]).

Definition 2.17 Suppose we have a tuple $\left(\mathscr{L},\left(V_{v}\right)_{v \in V(G)}\right)$ with $\mathscr{L}$ a line bundle of multidegree $w_{0}$ on $\widetilde{X}_{0}$, and each $V_{v}$ a $(r+1)$-dimensional space of global sections of $\mathscr{L}^{v}$ as in Proposition 2.15. For each pair $(e, v)$ in $\bar{G}$ where $v$ is a vertex of $e$, let $a_{0}^{e, v}, \ldots, a_{r}^{e, v}$ be the multivanishing sequence of $V_{v}$ along $D_{\bullet}^{e, v}$. Then $\left(\mathscr{L},\left(V_{v}\right)_{v \in V(G)}\right)$ is a limit linear series of multidegree $w_{0}$ with respect to $\left(w_{v}\right)_{v \in V(G)}$ on $\left(X_{0}, \boldsymbol{n}\right)$ if for any $e \in E(\bar{G})$ with vertices $v$ and $v^{\prime}$ we have

(i) for $l=0, \ldots, r$, if $a_{l}^{e, v}=\operatorname{deg} D_{j}^{e, v}$ with $j$ critical for $D_{\bullet}^{e, v}$, then $a_{r-l}^{e, v^{\prime}} \geq \operatorname{deg} D_{b_{v, v^{\prime}}-j^{\prime}}^{e, v^{\prime}}$;

(ii) there exists bases $s_{0}^{e, v}, \ldots, s_{r}^{e, v}$ of $V_{v}$ and $s_{0}^{e, v^{\prime}}, \ldots, s_{r}^{e, v^{\prime}}$ of $V_{v^{\prime}}$ satisfying the following conditions: 
(a) $\operatorname{ord}_{D_{\bullet}^{e, v}} s_{l}^{e, v}=a_{l}^{e, v}$ and $\operatorname{ord}_{D_{e}^{e, v^{\prime}}} s_{l}^{e, v^{\prime}}=a_{l}^{e, v^{\prime}}$ for $l=0,1, \ldots, r$;

(b) for all $j$ critical with respect to $D_{\bullet}^{e, v}$ and all $l$ such that $a_{l}^{e, v}=\operatorname{deg} D_{j}^{e, v}$ and $a_{r-l}^{e, v^{\prime}}=\operatorname{deg} D_{b_{v, v^{\prime}}-j}^{e, v^{\prime}}$, we have $\varphi_{j}^{e, v}\left(s_{l}^{e, v}\right)=s_{r-l}^{e, v^{\prime}}$.

Note that in part (b) $s_{l}^{e, v}$ (resp., $\left.s_{r-l}^{e, v^{\prime}}\right)$ is considered as a section in $V_{v}\left(-D_{j}^{e, v}\right)$ (resp., $\left.V_{v^{\prime}}\left(-D_{b_{v, v^{\prime}}}^{e, j}\right)\right)$, and $\varphi_{j}^{e, v}$ is as in Proposition 2.15.

Remark 2.18 Let $g_{j}=\#\left\{0 \leq l \leq r \mid a_{l}^{e, v}=\operatorname{deg} D_{j}^{e, v}\right.$ and $\left.a_{r-l}^{e, v^{\prime}}=\operatorname{deg} D_{b_{v, v^{\prime}}-j}^{e, v^{\prime}}\right\}$. Using the identification of the two linear spaces induced by $\varphi_{j}^{e, v}$, Definition 2.17(ii) is equivalent to the space

$$
\left(V_{v}\left(-D_{j}^{e, v}\right) / V_{v}\left(-D_{j+1}^{e, v}\right)\right) \cap\left(V_{v^{\prime}}\left(-D_{b_{v, v^{\prime}}-j}^{e, v^{\prime}}\right) / V_{v}\left(-D_{b_{v, v^{\prime}}-j+1}^{e, v^{\prime}}\right)\right)
$$

having dimension at least $g_{j}$.

\subsection{Limit Linear Series on Metrized Complexes}

In this subsection we introduce Amini and Baker's construction in [AB15] of metrized complexes and limit linear series on them. Suppose for now that $\kappa$ is algebraically closed. Recall that a metrized complex $\mathfrak{C}$ of curves over $\kappa$ consists of the following data: (1) a metric graph $\Gamma$ with underlying graph $G$; (2) for each vertex $v$ of $G$ a smooth curve $C_{v}$ over $\kappa$; (3) for each vertex $v$ of $G$, a bijection $e \mapsto x_{e}^{v}$ between the edges of $G$ incident to $v$ (recall that $G$ is assumed loopless in the beginning of this section) and a subset $A_{v}$ of $C_{v}(\kappa)$. For consistency of symbols we assume that $\Gamma$ is of integral edge lengths.

The geometric realization $|\mathfrak{C}|$ of $\mathfrak{C}$ is the union of the edges of $\Gamma$ and the collection of curves $C_{v}$, with each endpoint $v$ of an edge $e$ identified with $x_{e}^{v}$. The following is a geometric realization of a metrized complex which has rational $C_{v}$ for all $v \in V(G)$ and whose underlying graph $G$ is $K_{2}$.

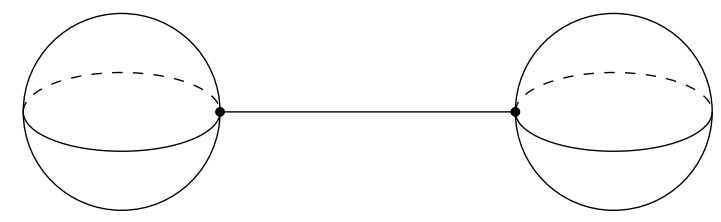

Example 2.19 Given a curve $X_{0}$ with dual graph $G$ and a chain structure $n$ on $G$, we can associate a metrized complex $\mathfrak{C}_{X_{0}, n}$ : let $\Gamma$ be the metric graph with underlying graph $G$ and edge lengths given by $n$, let $C_{v}$ be the component $Z_{v}$ and $x_{e}^{v}=P_{e}^{v}$ (hence $A_{v}=\mathcal{A}_{v}$ ). If the edge lengths are all 1 we denote by $\mathfrak{C}_{X_{0}}$ instead.

A divisor $\mathcal{D}$ on $\mathfrak{C}$ is a finite formal sum of points in $|\mathfrak{C}|$. Letting $\mathcal{D}=\sum_{x \in|\mathfrak{C}|} a_{x}(x)$, we can naturally associate a divisor $\mathcal{D}_{\Gamma}$ on $\Gamma$, called the $\Gamma$-part of $\mathcal{D}$, as well as, for each $v \in V(G)$, a divisor $\mathcal{D}_{v}$ on $C_{v}$ called the $C_{v}$-part of $\mathcal{D}$ as follows:

$$
\mathcal{D}_{v}=\sum_{x \in C_{v}(\kappa)} a_{x}(x) \text { and } \mathcal{D}_{\Gamma}=\sum_{x \in \Gamma \backslash V(G)} a_{x}(x)+\sum_{v \in V(G)} \operatorname{deg}\left(\mathcal{D}_{v}\right)(v) .
$$

Note that we have $\operatorname{deg} \mathcal{D}=\operatorname{deg} \mathcal{D}_{\Gamma}$. 
A nonzero rational function $\mathfrak{f}$ on $\mathfrak{C}$ is the data of a rational function $\mathfrak{f}_{\Gamma}$ (the $\Gamma$-part) on $\Gamma$ and nonzero rational functions $\mathfrak{f}_{v}$ (the $C_{v}$-part) on $C_{v}$ for each $v \in V(G)$. The divisor associated with $\mathfrak{f}$ is defined to be

$$
\operatorname{div}(\mathfrak{f}):=\sum_{x \in|\mathfrak{C}|} \operatorname{ord}_{x}(\mathfrak{f})(x)
$$

where $\operatorname{ord}_{x}(\mathfrak{f})$ is as follows: if $x \in \Gamma \backslash V(G)$, then $\operatorname{ord}_{x}(\mathfrak{f})=\operatorname{ord}_{x}\left(f_{\Gamma}\right)$; if $x \in C_{v}(\kappa) \backslash A_{v}$, then $\operatorname{ord}_{x}(\mathfrak{f})=\operatorname{ord}_{x}\left(\mathfrak{f}_{v}\right)$; if $x=P_{e}^{v} \in \mathcal{A}_{v}$, then $\operatorname{ord}_{x}(\mathfrak{f})=\operatorname{ord}_{x}\left(\mathfrak{f}_{v}\right)+\operatorname{slp}_{e, v}\left(\mathfrak{f}_{\Gamma}\right)$. In particular, the $\Gamma$-part of $\operatorname{div}(\mathfrak{f})$ is equal to $\operatorname{div}\left(\mathfrak{f}_{\Gamma}\right)$.

Similarly to the divisors on graphs we have the following definitions.

Definition 2.20 Suppose $\Gamma$ has integral edge lengths. A divisor $\mathcal{D}$ on $\mathfrak{C}$ is rational, integral, or edge-reduced if $\mathcal{D}_{\Gamma}$ is. Similarly, let $n$ be the chain structure on $G$ induced by $\Gamma$ and $w$ an admissible multidegree on $(G, n)$. Then $\mathcal{D}$ is of multidegree $w$ if $\mathcal{D}_{\Gamma}=$ $D_{w}$.

Definition 2.21 Divisors of the form $\operatorname{div}(\mathfrak{f})$ are called principal. Two divisors in $\operatorname{Div}(\mathfrak{C})$ are called linearly equivalent if they differ by a principal divisor. The rank $r_{\mathfrak{C}}(\mathcal{D})$ of a divisor $\mathcal{D}$ is the largest integer $r$ such that $\mathcal{D}-\mathcal{E}$ is linearly equivalent to an effective divisor for all effective divisor $\mathcal{E}$ of degree $r$ on $\mathfrak{C}$.

The definition of the rank of $\mathcal{D}$ can be refined by restricting the set of rational functions on $\mathfrak{C}$ that induce linear equivalence, as follows.

Definition 2.22 Suppose we are given, for each $v \in V(G)$, a non-empty $\kappa$-linear subspace $F_{v}$ of $K\left(C_{v}\right)$. Denote by $\mathcal{F}$ the collection of all $F_{v}$. We define the $\mathcal{F}$-rank $r_{\mathfrak{C}, \mathcal{F}}(\mathcal{D})$ of $\mathcal{D}$ to be the maximum integer $r$ such that for every effective divisor $\mathcal{E}$ of degree $r$, there is a nonzero rational function $\mathfrak{f}$ on $\mathfrak{C}_{\text {with }} \mathfrak{f}_{v} \in F_{v}$ for all $v \in V(G)$ such that $\mathcal{D}+\operatorname{div}(\mathfrak{f})-\mathcal{E} \geq 0$.

Note that by definition we always have $r_{\mathfrak{C}, \mathcal{F}}(\mathcal{D}) \leq r_{\mathfrak{C}}(\mathcal{D}) \leq r\left(\mathcal{D}_{\Gamma}\right)$. We are now able to define limit linear series on metrized complexes.

Definition 2.23 A limit linear series of degree $d$ and rank $r$ on $\mathfrak{C}$ is a (equivalence class of ) pair $(\mathcal{D}, \mathcal{H})$ consisting of a divisor $\mathcal{D}$ of degree $d$ and a collection $\mathcal{H}$ of $(r+1)$ dimensional subspaces $H_{v} \subset K\left(C_{v}\right)$ for all $v \in V(G)$, such that $r_{\mathfrak{C}, \mathcal{H}}(\mathcal{D})=r$. Two pairs $(\mathcal{D}, \mathcal{H})$ and $\left(\mathcal{D}^{\prime}, \mathcal{H}^{\prime}\right)$ are considered equivalent if there is a rational function $\mathfrak{f}$ on $\mathfrak{C}$ such that $\mathcal{D}^{\prime}=\mathcal{D}+\operatorname{div}(\mathfrak{f})$ and $H_{v}=H_{v}^{\prime} \cdot \mathfrak{f}_{v}$ for all $v \in V(G)$.

Definition 2.24 A limit linear series on $\mathfrak{C}$ is of multidegree $w_{0}$ if there is a representative $(\mathcal{D}, \mathcal{H})$ such that $\mathcal{D}$ is so.

Let $X$ be a smooth curve over $\widetilde{K}$. Recall that a strongly semistable model for $X$ is a flat and integral proper relative curve over $\widetilde{R}$ whose generic fiber is isomorphic to $X$ and whose special fiber is a curve in our setting (or a strongly semistable curve over $\kappa$ as in $[A B 15, \S 4.1])$. Given a strongly semistable model $\mathfrak{X}$, again there is an associated metrized complex $\mathfrak{C X}$ where the underlying graph $G$ is the dual graph of 
the special fiber $X_{0}=\overline{\mathfrak{X}}$ and $C_{v}$ is the component $Z_{v}$ of $X_{0}$ and $x_{e}^{v}=P_{e}^{v}$. Moreover, the length $l(e)$ of $e \in E(\Gamma)=E(G)$ is $\operatorname{val}\left(\omega_{e}\right)$ for some $w_{e} \in \widetilde{R}$ such that the local equation of $\mathfrak{X}$ at the node $P_{e}$ of $e$ is $x y-\omega_{e}$. Equivalently, consider the natural reduction map red: $X(\widetilde{K}) \rightarrow X_{0}(\kappa)$ induced by the bijection between $X(\widetilde{K})$ and $\mathfrak{X}(\widetilde{R})$; this extends to a map red: $X^{\text {an }} \rightarrow X_{0}$ and $l(e)$ is the modulus of the open (analytic) annulus $\operatorname{red}^{-1}\left(P_{e}\right)$ in $X^{\text {an }}$, where $X^{\text {an }}$ is the Berkovich analytification of $X$. Note that any metrized complex with edge lengths contained in $\operatorname{val}(\widetilde{K})$ can be constructed from strongly semistable models ( $c f$. [AB15, Theorem 4.1]).

There is a canonical embedding of $\Gamma$ into $X^{\text {an }}$ as well as a canonical retraction map $\tau: X^{\text {an }} \rightarrow \Gamma$, which induces by linearity a specialization map $\tau_{\star}: \operatorname{Div}(X) \rightarrow \operatorname{Div}(\Gamma)$ which maps $X(\widetilde{K})$ to the set of rational points of $\Gamma$. For $P \in X(\widetilde{K})$, if $\tau_{*}(P)=v \in$ $V(G)$, then $\operatorname{red}(P)$ is a nonsingular closed point of $X_{0}$ in $C_{v}$. We thus have the specialization map of divisors $\tau_{*}^{\mathfrak{C X}}: \operatorname{Div}(X) \rightarrow \operatorname{Div}(\mathfrak{C X})$ given by the linear extension of

$$
\tau_{*}^{\mathfrak{C X}}(P)= \begin{cases}\tau_{*}(P) & \tau_{*}(P) \notin V(G) \\ \operatorname{red}(P) & \tau_{*}(P) \in V(G) .\end{cases}
$$

On the other hand, let $x \in X^{\text {an }}$ be a point of type 2. The completed residue field $\overline{\mathcal{H}(x)}$ of $x$ has transcendence degree one over $\kappa$ and corresponds to a curve $C_{x}$ over $\kappa$. Given a nonzero rational function $f$ on $X$, choose $c \in \widetilde{K}^{\times}$such that $|f(x)|=|c|$. We denote by $f_{x}$ the image of $c^{-1} f$ in $K\left(C_{x}\right) \cong \widetilde{\mathcal{H}(x)}$, which is well defined up to scaling by $\kappa^{\times}$. We call $f_{x}$ the normalized reduction of $f$. Note that the normalized reduction of a $\widetilde{K}$-vector space $V$ is a $\kappa$-vector space of the same dimension, whereas the normalized reduction of a basis of $V$ is not necessarily a basis of the normalized reduction of $V$. See [AB15, Lemma 4.3] for details.

Definition 2.25 Given $X$ and $\mathfrak{X}$ as above, the specialization $\tau_{*}^{\mathfrak{C X}}(f)$ of $0 \neq f \in$ $K(X)$ is a nonzero rational function on $\mathfrak{C X}$ whose $\Gamma$-part is the restriction to $\Gamma$ of the piecewise linear function $F=\log |f|$ on $X^{\text {an }}$ and whose $Z_{v}$-part is (up to multiplication by $\kappa^{\times}$) the normalized reduction $f_{x_{v}}$ in which $x_{v}$, whose completed residue field is identified with the function field of $Z_{v}$, is the image of $v$ under the embedding $\Gamma \hookrightarrow$ $X^{\text {an }}$ mentioned above. The specialization $\tau_{*}^{\mathfrak{C X}}(V)$ of a linear subspace $V$ of $K(X)$ is a collection $\left\{F_{v}\right\}_{v \in V(G)}$ of space of rational functions on $Z_{v}$, where $F_{v}=\left\{f_{x_{v}} \mid f \in V\right\}$. We denote by $\tau_{* v}^{\mathfrak{C X}}$ the $Z_{v}$-part of $\tau_{*}^{\mathfrak{C X}}$, or equivalently the normalized reduction to $\overline{\mathcal{H}\left(x_{v}\right)}$.

Note that $\tau_{* v}^{\mathfrak{C X}}(V)$ is a linear space of the same dimension as $V$ as mentioned above. Similar to the classical case, we have that the specialization of a linear series is a limit linear series.

Theorem 2.26 ([AB15, Theorem 5.9]) Let $X, \mathfrak{X}$ be as above. Let $D$ be a divisor on $X$ and let $\left(\mathscr{O}_{X}(D), V\right)$ be $a \mathfrak{g}_{d}^{r}$ on $X$, where $V \subset H^{0}\left(X, \mathscr{O}_{X}(D)\right) \subset K(X)$. Then the pair $\left(\tau_{*}^{\mathfrak{C X}}(D), \tau_{*}^{\mathfrak{C X}}(V)\right)$ is a limit $\mathfrak{g}_{d}^{r}$ on $\mathfrak{C X}$.

We end the section with the definition of smoothability of limit linear series on metrized complexes. 
Definition 2.27 A limit linear series $(\mathcal{D}, \mathcal{H})$ of degree $d$ and rank $r$ on a metrized complex $\mathfrak{C}$ over $\kappa$ is called smoothable if there exists a smooth proper curve $X$ over $\widetilde{K}$ such that $\mathfrak{C}=\mathfrak{C} \mathfrak{X}$ for some strongly semistable model $\mathfrak{X}$ of $X$ and $(\mathcal{D}, \mathcal{H})$ arises from the specialization of a $\mathfrak{g}_{d}^{r}$ on $X$.

\section{The Weak Glueing Condition}

In this section we introduce the concept of pre-limit linear series on a curve $X_{0}$ of pseudocompact type with given chain structure $\boldsymbol{n}$ (on the dual graph), and a forgetful map from the space of pre-limit linear series on $\left(X_{0}, n\right)$ to the set of limit linear series on $\mathfrak{C}_{X_{0}, n}$, derived from [Oss17].

We show that this map is bijective onto the set of $(\mathcal{D}, \mathcal{H})$ with integral $\mathcal{D}$. By this we can extend the weak glueing condition on curves, which will be defined in the beginning of this section, to metrized complexes, and transfer the smoothing problems on metrized complexes to those on curves. To be consistent with Section 2.3, we assume in this section that $\kappa$ is algebraically closed. We use the following notation.

Notation 3.1 Let $X_{0}$ be a curve over $\kappa$ of pseudocompact type, $G$ the dual graph of $X_{0}$ and $\bar{G}$ the tree obtained from $G$ as in $\$ 1.1$. Let $n$ be a chain structure on $G$ and $\Gamma$ the induced metric graph. Take $w_{0}$ an admissible multidegree of total degree $d$ on $(G, \boldsymbol{n})$. Choose also a tight tuple $\left(w_{v}\right)_{v \in V(G)}$ of admissible multidegrees in $V\left(G\left(w_{0}\right)\right)$. Let $d_{v}$ be the coefficient of $D_{w_{v}}$ at $v$. Let $\widetilde{X}_{0}$ be the nodal curve corresponding to $n$ as constructed in Section 2.2 (after Definition 2.12) and $\widetilde{G}$ the dual graph of $\widetilde{X}_{0}$. Let $\mathfrak{C}_{X_{0}, n}$ and $\mathfrak{C}_{\widetilde{X}_{0}}$ be the induced metrized complexes as in Example 2.19. Given an enriched structure on $\widetilde{X}_{0}$, for a line bundle $\mathscr{L}$ on $\widetilde{X}_{0}$ recall that $\mathscr{L}^{v}=\left.\mathscr{L}_{w_{v}}\right|_{Z_{v}}$ is constructed as in Proposition 2.15.

With the notation above, we start by recalling pre-limit linear series on $\left(X_{0}, n\right)$ and the weak glueing condition.

Definition 3.2 A pre-limit linear series of rank $r$ with respect to the admissible multidegrees $\left(w_{v}\right)_{v}$ on $\left(X_{0}, n\right)$ is a tuple $\left(\mathscr{L}_{v}, V_{v}\right)_{v \in V(G)}$ of line bundles $\mathscr{L}_{v}$ on $Z_{v}$ of degree $d_{v}$ and linear spaces $V_{v} \subset H^{0}\left(Z_{v}, \mathscr{L}_{v}\right)$ of dimension $r+1$ such that the multivanishing sequence $a_{\bullet}^{e, v}$ of $V_{v}$ along the twisting divisors $D_{\bullet}^{e, v}$ satisfies condition (i) of Definition 2.17. Given an enriched structure, we say a pre-limit linear series lifts to a limit linear series if there exists a line bundle $\mathscr{L}$ on $\widetilde{X}_{0}$ of multidegree $w_{0}$ such that $\mathscr{L}_{v}=\mathscr{L}^{v}$, and the tuple $\left(\mathscr{L},\left(V_{v}\right)_{v}\right)$ is a limit linear series as in Definition 2.17.

For an edge $e \in E(\bar{G})$ with incident vertices $v$ and $v^{\prime}$ and a $j$ critical for $D_{\bullet}^{e, v}$, suppose $D_{j+1}^{e, v}-D_{j}^{e, v}=P_{e_{1}}^{v}+\cdots+P_{e_{m}}^{v}$, where $e_{i} \in E(G)$ lies over $e$. We have an isomorphism

$$
\psi: H^{0}\left(Z_{v}, \mathscr{L}_{v}\left(-D_{j}^{e, v}\right) / \mathscr{L}_{v}\left(-D_{j+1}^{e, v}\right)\right) \longrightarrow \kappa^{m}
$$

given by $\psi(s)=\left(s\left(P_{e_{1}}^{v}\right), \ldots, s\left(P_{e_{m}}^{v}\right)\right)$, which is unique up to coordinate-wise scaling. This induces an action of the $m$-dimensional torus on $\mathscr{L}_{v}\left(-D_{j}^{e, v}\right) / \mathscr{L}_{v}\left(-D_{j+1}^{e, v}\right)$ that is 
independent of $\psi$. Similarly, we have a torus action on

$$
\mathscr{L}_{v^{\prime}}\left(-D_{b_{v, v^{\prime}}-j}^{e, v^{\prime}}\right) / \mathscr{L}_{v^{\prime}}\left(-D_{b_{v, v^{\prime}}-j+1}^{e, v^{\prime}}\right)
$$

and $\varphi_{j}^{e, v}$ (as in Proposition 2.15) respects torus orbits.

Definition 3.3 We say that a pre-limit linear series $\left(\mathscr{L}_{v}, V_{v}\right)_{v \in V(G)}$ satisfies the weak glueing condition if for all $e \in \bar{G}$ with incident vertices $v$ and $v^{\prime}$, and for all $j$ critical for $D_{\bullet}^{e, v}$, there exists subspaces

$$
\begin{gathered}
W_{v, j} \subset V_{v}\left(-D_{j}^{e, v}\right) / V_{v}\left(-D_{j+1}^{e, v}\right), \\
W_{v^{\prime}, b_{v, v^{\prime}}-j} \subset V_{v^{\prime}}\left(-D_{b_{v, v^{\prime}}}^{e, j}\right) / V_{v^{\prime}}\left(-D_{b_{v, v^{\prime}}}^{e, j+1}\right),
\end{gathered}
$$

both of which have dimension $g_{j}$, such that for all torus orbits

$$
\begin{gathered}
T_{v} \subset \mathscr{L}_{v}\left(-D_{j}^{e, v}\right) / \mathscr{L}_{v}\left(-D_{j+1}^{e, v}\right), \\
T_{v^{\prime}}=\varphi_{j}^{e, v}\left(T_{v}\right) \subset \mathscr{L}_{v^{\prime}}\left(-D_{b_{v, v^{\prime}}-j}^{e, v^{\prime}}\right) / \mathscr{L}_{v^{\prime}}\left(-D_{b_{v, v^{\prime}}-j+1}^{e, v^{\prime}}\right),
\end{gathered}
$$

we have

$$
\operatorname{dim}\left(T_{v} \cap W_{v, j}\right)=\operatorname{dim}\left(T_{v^{\prime}} \cap W_{v^{\prime}, b_{v, v^{\prime}}-j}\right) .
$$

Here $g_{j}$ is defined in Remark 2.18.

Remark 3.4 In the above definition it is enough to check that for all torus orbits $T_{v}$ we have that $T_{v} \cap W_{v, j}$ is nonempty if and only if $T_{v^{\prime}} \cap W_{v^{\prime}, b_{v, v^{\prime}}-j}$ is nonempty: let $T_{v}=\left\{\left(x_{1}, \ldots, x_{m}\right) \mid x_{i} \neq 0\right.$ if and only if $\left.i \in I\right\}$ for some $I \subset\{1, \ldots, m\}$ and $\operatorname{dim}\left(T_{v} \cap\right.$ $\left.W_{v, j}\right)=l$. For $s=\left(a_{1}, \ldots, a_{m}\right) \in \mathscr{L}_{v}\left(-D_{j}^{e, v}\right) / \mathscr{L}_{v}\left(-D_{j+1}^{e, v}\right)$, denote $I_{s}=\left\{i \mid a_{i} \neq\right.$ $0\}$. We can find $s_{1}, \ldots, s_{l} \in T_{v} \cap W_{v, j}$ that are linearly independent. Taking linear combinations of $\left\{s_{i}\right\}$ gives $t_{1}, \ldots, t_{l} \in W_{v, j}$ such that $I_{t_{1}} \mp I_{t_{2}} \mp \cdots \mp I_{t_{l}}=I$. Now let $T_{v^{\prime}}^{i}=\left\{\left(x_{1}^{\prime}, \ldots, x_{m}^{\prime}\right) \mid x_{i}^{\prime} \neq 0\right.$ if and only if $\left.i \in I_{t_{i}}\right\} \subset \bar{T}_{v^{\prime}}$. We have that $T_{v^{\prime}}^{l}=T_{v^{\prime}}$ and $W_{v^{\prime}, b_{v, v^{\prime}}-j} \cap T_{v^{\prime}}^{i} \neq \varnothing$ by assumption, which implies that $\operatorname{dim}\left(T_{v^{\prime}} \cap W_{v^{\prime}, b_{v, v^{\prime}}-j}\right) \geq l=$ $\operatorname{dim}\left(T_{v} \cap W_{v, j}\right)$, since $W_{v^{\prime}, b_{v, v}-j}$ is a $\kappa$-vector space. The same argument shows that $\operatorname{dim}\left(T_{v} \cap W_{v, j}\right) \geq \operatorname{dim}\left(T_{v^{\prime}} \cap W_{v^{\prime}, b_{v, v^{\prime}}-j}\right)$, hencedim $\left(T_{v} \cap W_{v, j}\right)=\operatorname{dim}\left(T_{v^{\prime}} \cap W_{v^{\prime}, b_{v, v^{\prime}}}\right)$.

Example 3.5 Note that $g_{j} \leq \operatorname{deg} D_{j+1}^{e, v}-\operatorname{deg} D_{j}^{e, v}$. If $g_{j}=\operatorname{deg} D_{j+1}^{e, v}-\operatorname{deg} D_{j}^{e, v}$, then the weak glueing condition is trivial, since in this case we have

$$
\begin{aligned}
W_{v, j} & =\mathscr{L}_{v}\left(-D_{j}^{e, v}\right) / \mathscr{L}_{v}\left(-D_{j+1}^{e, v}\right), \\
W_{v^{\prime}, b_{v, v^{\prime}}-j} & =\mathscr{L}_{v^{\prime}}\left(-D_{b_{v, v^{\prime}}-v^{\prime}}^{e, j}\right) / \mathscr{L}_{v^{\prime}}\left(-D_{b_{v, v^{\prime}}-v^{\prime}+1}^{e}\right) .
\end{aligned}
$$

On the other hand, if $g_{j}=1$, then the weak glueing condition is equivalent to the existence of

$$
s_{v} \in V_{v}\left(-D_{j}^{e, v}\right) / V_{v}\left(-D_{j+1}^{e, v}\right) \quad \text { and } \quad s_{v^{\prime}} \in V_{v^{\prime}}\left(-D_{b_{v, v^{\prime}}-j}^{e, v^{\prime}}\right) / V_{v^{\prime}}\left(-D_{b_{v, v^{\prime}}-j+1}^{e, v^{\prime}}\right)
$$

such that $s_{v}$ vanishes at $P_{v}^{\tilde{e}}$ if and only if $s_{v^{\prime}}$ vanishes at $P_{v^{\prime}}^{\tilde{e}}$, where $P_{v}^{\tilde{e}}$ runs over the support of $D_{j+1}^{e, v}-D_{j}^{e, v}$.

See also Example 4.11 for a concrete case of a pre-limit linear series (as well as a limit linear series on $\mathfrak{C}_{X_{0}, n}$ ) that does not satisfy the weak glueing condition. 
Proposition 3.6 A pre-limit linear series that lifts to a limit linear series must satisfy the weak glueing condition.

Proof This follows directly from Remark 2.18.

Let $P_{d}^{r}\left(X_{0}, \boldsymbol{n},\left(w_{v}\right)_{v}\right)$ be the space ${ }^{1}$ of pre-limit $\mathfrak{g}_{d}^{r}$ s with respect to $\left(w_{v}\right)_{v}$ on $\left(X_{0}, \boldsymbol{n}\right)$, and $\mathfrak{G}_{d}^{r}\left(\mathfrak{C}_{X_{0}, n}\right)$ be the set of limit $\mathfrak{g}_{d}^{r}$ s on $\mathfrak{C}_{X_{0}, n}$. Given $w, w^{\prime} \in V\left(G\left(w_{0}\right)\right)$, we denote by $D_{w, w^{\prime}}^{v}$ the divisor on $Z_{v}$ obtained by twisting from $w$ to $w^{\prime}$. More precisely, if $D_{w^{\prime}}=$ $D_{w}+\operatorname{div}(f)$ where $f$ is a piecewise linear function on $\Gamma$, then $D_{w, w^{\prime}}^{v}=\sum \operatorname{slp}_{e, v}(f) P_{e}^{v}$ where the summation is taken over all edges in $G$ that are incident to $v$. In other words, if $P\left(w, v_{1}, \ldots, v_{m}\right)=\left(w_{1}, \mu_{1}\right), \ldots,\left(w_{m+1}, \mu_{m+1}\right)$ is a path in $G\left(w_{0}\right)$ from $w$ to $w^{\prime}$, let $S \subset\{1, \ldots, m\}$ consist of $i$ such that $v_{i}(\neq v)$ is adjacent to $v$, and for $i \in S$ let $e_{i} \in E(\bar{G})$ be the edge connecting $v$ and $v_{i}$. Then ([Oss17, Notation 4.7])

$$
D_{w, w^{\prime}}^{v}=\sum_{i \in S} \sum_{\substack{\tilde{e} \in E(G) \text { over } e_{i} \\ \mu_{i+1}(\tilde{e})=0}} P_{\tilde{e}}^{v}-\sum_{i: v_{i}=v} \sum_{\substack{\tilde{e} \in E(G) \text { incident on } v \\ \mu_{i}(\tilde{e})=0}} P_{\tilde{e}}^{v} .
$$

Definition 3.7 We define a map $\mathfrak{F}_{\left(w_{v}\right)_{v}}: P_{d}^{r}\left(X_{0}, n,\left(w_{v}\right)_{v}\right) \rightarrow \mathfrak{G}_{d}^{r}\left(\mathfrak{C}_{X_{0}, n}\right)$ as follows: given a tuple $\left(\mathscr{L}_{v}, V_{v}\right)_{v} \in P_{d}^{r}\left(X_{0}, n,\left(w_{v}\right)_{v}\right)$, for each $v \in V(G)$, choose nonzero $s_{v} \in$ $V_{v}$. Fix $w \in V\left(G\left(w_{0}\right)\right)$. Take $\mathcal{D} \in \operatorname{Div}\left(\mathfrak{C}_{X_{0}, n}\right)$ of multidegree $w$ such that $\mathcal{D}_{v}=$ $\operatorname{div}^{0}\left(s_{v}\right)-D_{w, w_{v}}^{v}$ for all $v$ and a collection $\mathcal{H}=\left(H_{v}\right)_{v}$ of rational functions with $H_{v}=\left\{\frac{s}{s_{v}}: s \in V_{v}\right\}$. Then we define $\mathfrak{F}_{\left(w_{v}\right)_{v}}\left(\left(\mathscr{L}_{v}, V_{v}\right)\right)=(\mathcal{D}, \mathcal{H})$. We sometimes write $\mathfrak{F}$ instead of $\mathfrak{F}_{\left(w_{v}\right)_{v}}$ when $\left(w_{v}\right)_{v}$ is specified.

Note that by [Oss17, Propostions 4.12 and 5.9] the map $\mathfrak{F}_{\left(w_{v}\right)_{v}}$ is well defined; i.e., $\mathfrak{F}_{\left(w_{v}\right)_{v}}\left(\left(\mathscr{L}_{v}, V_{v}\right)_{v}\right)$ is a limit $\mathfrak{g}_{d}^{r}$ on $\mathfrak{C}_{X_{0}, n}$ for all $\left(\mathscr{L}_{v}, V_{v}\right)_{v} \in P_{d}^{r}\left(X_{0}, n,\left(w_{v}\right)_{v}\right)$, and it is independent of choices of $s_{v}$ and $w$. We next show that $\mathfrak{F}_{\left(w_{v}\right)}$ is a bijection onto the set of limit $\mathfrak{g}_{d}^{r}$ s on $\mathfrak{C}_{X_{0}, n}$ of multidegree $w_{0}$.

Given $(\mathcal{D}, \mathcal{H})$ a limit $\mathfrak{g}_{d}^{r}$ on $\mathfrak{C}_{X_{0}, n}$ with $\mathcal{D}_{\Gamma}=D_{w_{0}}$. Recall that $w_{v}^{\text {red }} \in V\left(G\left(w_{0}\right)\right)$ is the admissible multidegree such that $D_{w_{v}^{\text {red }}}$ is $v$-reduced on $\Gamma$. We have that $\left(w_{v}^{\text {red }}\right)_{v}$ is a tight tuple by Proposition 2.10. Let $D_{v}^{\text {red }}:=D_{w_{0}, w_{v}^{\text {red }}}^{v}$. We have the following lemma.

Lemma 3.8 For any $v \in V(G)$, we have $H_{v} \subset H^{0}\left(Z_{v}, \mathscr{O}_{Z_{v}}\left(\mathcal{D}_{v}+D_{v}^{\text {red }}\right)\right)$.

Proof Take $v_{0} \in V(G)$ and a rational function $f$ on $\Gamma$ such that $D_{w_{v_{0}}^{\text {red }}}=D_{w_{0}}+\operatorname{div}(f)$. Take a rational function $\mathfrak{f}$ on $\mathfrak{C}_{X_{0}, n}$ such that $\mathfrak{f}_{\Gamma}=f$ and $\mathfrak{f}_{v}=1$ for all $v \in V(G)$. Let $\mathcal{D}^{\prime}=\mathcal{D}+\operatorname{div}(\mathfrak{f})$. We then have $\mathcal{D}_{v_{0}}^{\prime}=\mathcal{D}_{v_{0}}+D_{v_{0}}^{\text {red }}$ and $\left(\mathcal{D}^{\prime}, \mathcal{H}\right)$ is also a limit $\mathfrak{g}_{d}^{r}$, and $\mathcal{D}_{\Gamma}^{\prime}$ is the $v_{0}$-reduced divisor on $\Gamma$ that is linearly equivalent to $\mathcal{D}_{\Gamma}$.

Take $r$ general points $P_{1}, \ldots, P_{r} \in Z_{v_{0}}$; there is a $\mathfrak{h} \in \operatorname{Rat}\left(\mathfrak{C}_{X_{0}, n}\right)$ such that $\mathcal{D}^{\prime}+$ $\operatorname{div}(\mathfrak{h})-P_{1}-\cdots-P_{r} \geq 0$ and that $\mathfrak{h}_{v} \in H_{v}$ for all $v$. It follows that $\mathcal{D}_{\Gamma}^{\prime}+\operatorname{div}\left(\mathfrak{h}_{\Gamma}\right)-r v_{0}$ is an

\footnotetext{
${ }^{1}$ Here is a description of $P_{d}^{r}\left(X_{0}, n,\left(w_{v}\right)_{v}\right)$ : take non-negative sequences $a_{\bullet}^{e, v}$ for each adjacent pair $(e, v)$ in $\bar{G}$ satisfying condition (i) of Definition 2.17 , let $G_{d}^{r}\left(Z_{v},\left(D_{\bullet}^{e, v}, a_{\bullet}^{e, v}\right)_{e}\right)$ be the space of $\mathfrak{g}_{d_{v}}^{r}$ s on $Z_{v}$ having multivanishing sequence at least $a_{\bullet}^{e, v}$ along $D_{\bullet}^{e, v}$ for all $e$ incident to $v$. Then $P_{d}^{r}\left(X_{0}, n,\left(w_{v}\right)_{v}\right)$ is the union over all such $\left(a_{\bullet}^{e, v}\right)_{e, v}$ of $\prod_{v \in V(G)} G_{d_{v}}^{r}\left(Z_{v},\left(D_{\bullet}^{e, v}, a_{\bullet}^{e, v}\right)_{e}\right)$ in $\prod_{v \in V(G)} G_{d_{v}}^{r}\left(Z_{v}\right)$, where $G_{d_{v}}^{r}\left(Z_{v}\right)$ is the space of $\mathfrak{g}_{d_{v}}^{r}$ s on $Z_{v}$.
} 
effective divisor on $\Gamma$. We denote this divisor by $D$ and hence $\mathcal{D}_{\Gamma}^{\prime}=D+\operatorname{div}\left(-\mathfrak{h}_{\Gamma}\right)+r v_{0}$. We claim that $\operatorname{slp}_{e, v_{0}}\left(\mathfrak{h}_{\Gamma}\right) \leq 0$ for all $e \in E(G)$ incident to $v_{0}$.

Suppose there is a $e_{0} \in E(\bar{G})$ incident to $v_{0}$ and $\tilde{e}_{0} \in E(G)$ lying over $e_{0}$ such that $\operatorname{slp}_{\tilde{e}_{0}, v_{0}}\left(\mathfrak{h}_{\Gamma}\right)>0$ (or equivalently $\operatorname{slp}_{\tilde{e}_{0}, v_{0}}\left(-\mathfrak{h}_{\Gamma}\right)<0$ ). Let $v_{1}$ be the other vertex of $e_{0}$. Since $\mathcal{D}_{\Gamma}^{\prime}$ is $v_{0}$-reduced, for all $x \in \Gamma \backslash V(G)$ we have $\operatorname{ord}_{x}\left(-\mathfrak{h}_{\Gamma}\right) \leq 1$. Therefore, for all $\tilde{e} \in E(G)$ lying over $e_{0}$ either $\operatorname{slp}_{\tilde{e}, v_{1}}\left(-\mathfrak{h}_{\Gamma}\right)>0$ or there is a point $x \in \bar{e}^{-\circ}$ such that $\operatorname{ord}_{x}\left(-\mathfrak{h}_{\Gamma}\right)>0$, where $\bar{e} \in E(\Gamma)$ is the edge induced by $\tilde{e}$. Now Dhar's algorithm ([Luo11]) implies that there must exist an edge $e_{1} \in E(\bar{G}) \backslash\left\{e_{0}\right\}$ incident to $v_{1}$ and $\tilde{e}_{1} \epsilon$ $E(G)$ lying over $e_{1}$ such that $\operatorname{slp}_{\tilde{e}_{1}, v_{1}}\left(-\mathfrak{h}_{\Gamma}\right)<0$. Inductively we end up with a sequence of distinct vertices $v_{0}, v_{1}, \ldots$ and edges $\tilde{e}_{1}, \tilde{e}_{2}, \cdots \in E(G)$, lying over $e_{1}, e_{2}, \cdots \in E(\bar{G})$ respectively, such that $e_{i}$ is incident to $v_{i}$ and $v_{i+1}$ and that $\operatorname{slp}_{\tilde{e}_{i}, v_{i}}\left(-\mathfrak{h}_{\Gamma}\right)<0$. This sequence must be finite, since $\bar{G}$ is a tree, which provides a contradiction.

It follows that $\mathcal{D}_{v_{0}}^{\prime}+\operatorname{div}\left(\mathfrak{h}_{v_{0}}\right)-P_{1}-\cdots-P_{r} \geq 0$. Now the generality of $P_{i}$ implies that $H^{0}\left(Z_{v_{0}}, \mathscr{O}_{Z_{v_{0}}}\left(\mathcal{D}_{v_{0}}^{\prime}\right)\right) \cap H_{v_{0}}$ has dimension at least $r+1$, hence we have $H_{v_{0}} \subset$ $H^{0}\left(Z_{v_{0}}, \mathscr{O}_{Z_{v_{0}}}\left(\mathcal{D}_{v_{0}}^{\prime}\right)\right)=H^{0}\left(Z_{v_{0}}, \mathscr{O}_{Z_{v_{0}}}\left(\mathcal{D}_{v_{0}}+D_{v_{0}}^{\text {red }}\right)\right)$.

Let $\mathfrak{G}_{d}^{r}\left(\mathfrak{C}_{X_{0}, n}, w_{0}\right)$ be the set of limit $\mathfrak{g}_{d}^{r}$ s of multidegree $w_{0}$. We first show the bijectivity of $\mathfrak{F}$ for the tuple $\left(w_{v}^{\text {red }}\right)_{v}$.

Theorem 3.9 The map $\mathfrak{F}_{\left(w_{v}^{\text {red }}\right)_{v}}$ is a bijection onto $\mathfrak{G}_{d}^{r}\left(\mathfrak{C}_{X_{0}, n}, w_{0}\right)$.

Proof It is easy to check that $\mathfrak{F}$ is injective. We next show surjectivity. Take $(\mathcal{D}, \mathcal{H})$ $\in \mathfrak{G}_{d}^{r}\left(\mathfrak{C}_{X_{0}, n}\right)$. According to Lemma 3.8, we have $H_{v} \subset H^{0}\left(Z_{v}, \mathscr{O}_{Z_{v}}\left(\mathcal{D}_{v}+D_{v}^{\text {red }}\right)\right)$. We next show that $\left(\mathscr{L}_{v}, V_{v}\right)_{v}=\left(\mathscr{O}_{Z_{v}}\left(\mathcal{D}_{v}+D_{v}^{\text {red }}\right), H_{v}\right)_{v}$ is a pre-limit $\mathfrak{g}_{d}^{r}$ on $\left(X_{0}, \boldsymbol{n}\right)$ by extending the method of the proof of [AB15, Theorem 5.4]. Note that afterwards straightforward calculation shows that $\mathfrak{F}\left(\left(\mathscr{L}_{v}, V_{v}\right)_{v}\right)=(\mathcal{D}, \mathcal{H})$.

We can assume that $\mathcal{D}_{\Gamma}=D_{w_{0}}$. Fix an edge $e \in E(\bar{G})$. Let $e_{1}, \ldots, e_{m}$ be the edges of $G$ lying over $e$ and let $v_{1}, v_{2}$ be the vertices of $e$. For simplicity let $\mathcal{D}_{j}=\mathcal{D}_{v_{j}}, D_{j}=$ $D_{v_{j}}^{\text {red }}, H_{j}=H_{v_{j}}, Z_{j}=Z_{v_{j}}, \mathscr{L}_{j}=\mathscr{L}_{v_{j}}$, and $w_{j}=w_{v_{j}}^{\text {red }}$ for $j=1,2$. Denote by $P_{i}^{j}=P_{e_{i}}^{v_{j}}$ the points on $Z_{j}$ corresponding to $e_{i}$ and by $\mathcal{A}_{j}=\mathcal{A}_{e}^{v_{j}}$ the set of points $P_{i}^{j}$. Suppose $e$ is directed by $v_{1} \rightarrow v_{2}$ and $w_{0}=\left(w_{G}, \mu\right)$. Denote $x_{i}=\mu\left(e_{i}\right)$ for $1 \leq i \leq m$.

Suppose $w_{2}$ is equal to $w_{1}$ twisting $b$ times at $\left(e, v_{1}\right)$. We show that the condition about multivanishing sequences (Definition 2.17(i)) is satisfied for our fixed $e$, which completes our proof.

Let $a_{0}^{e, v_{j}}, \ldots, a_{r}^{e, v_{j}}$ be the multivanishing sequences of $H_{j}$ along $D_{\bullet}^{e, v_{j}}$ as in Definition 2.17. For $l=0,1, \ldots, r$ we claim that there is an $s$ such that $a_{l}^{e, v_{1}} \geq D_{s}^{e, v_{1}}$ and $a_{r-l}^{e, v_{2}} \geq D_{b-s}^{e, v_{2}}$.

Take effective divisors

$$
\left(E_{1}, E_{2}\right)=\left(\sum_{1 \leq k \leq r-l} Q_{k}, \sum_{r-l+1 \leq k \leq r} Q_{k}\right) \in\left(Z_{1}^{(r-l)}, Z_{2}^{(l)}\right)
$$

where $Z_{j}^{(l)}$ is the $l$-fold symmetric product. For $v \in V(G)$, denote by $E(v)$ the set of edges of $G$ incident to $v$. Let $F_{E_{1}, E_{2}}$ be the set of divisors $\mathcal{E}$ on $\mathfrak{C}_{X_{0}, n}$ of the form

$$
\mathcal{E}=\sum_{j=1,2} \sum_{e^{\prime} \in E\left(v_{j}\right)} \operatorname{slp}_{e^{\prime}, v_{j}}\left(\mathfrak{f}_{\Gamma}\right) P_{e^{\prime}}^{v_{j}}
$$


where $\mathfrak{f}$ is a rational function on $\mathfrak{C}$ such that $\mathfrak{f}_{v} \in H_{v}$ for all $v \in V(G)$ and $\operatorname{div}(\mathfrak{f})+\mathcal{D}-$ $\left.\left(E_{1}+E_{2}\right) \geq 0\right\}$. Then the set

$$
F=\bigcup_{\left(E_{1}, E_{2}\right)} F_{E_{1}, E_{2}}
$$

is finite. For $D_{0} \in F$, let $S_{D_{0}}$ be the subset of $Z_{1}^{(r-l)} \times Z_{2}^{(l)}$ defined by all collections of effective divisors $\left(E_{1}, E_{2}\right)$ such that $D_{0} \in F_{E_{1}, E_{2}}$. We have the following:

(i) Each $S_{D_{0}}$ is Zariski closed in $Z_{1}^{(r-l)} \times Z_{2}^{(l)}$. Indeed, $S_{D_{0}}$ is of the form $\Lambda_{1} \times \Lambda_{2}$, where $\Lambda_{1}\left(\right.$ resp. $\Lambda_{2}$ ) is the Zariski closed subset of $Z_{1}^{(r-l)}$ (resp., $Z_{2}^{(l)}$ ) consisting of all divisors $E_{1}$ (resp., $E_{2}$ ) such that there exists $f_{1} \in H_{1}$ (resp., $f_{2} \in H_{2}$ ) such that $\operatorname{div}\left(f_{1}\right)+\left.\left(D_{0}+\mathcal{D}\right)\right|_{Z_{1}}-E_{1} \geq 0\left(\right.$ resp., $\left.\operatorname{div}\left(f_{2}\right)+\left.\left(D_{0}+\mathcal{D}\right)\right|_{Z_{2}}-E_{2} \geq 0\right)$.

(ii) $\cup_{D_{0} \in F} S_{D_{0}}=Z_{1}^{(r-l)} \times Z_{2}^{(l)}$.

Therefore, we can find a $D_{0}$ such that $S_{D_{0}}=Z_{1}^{(r-l)} \times Z_{2}^{(l)}$. Now for any choices of $Q_{1}, \ldots, Q_{r-l} \in Z_{1}$ and $Q_{r-l+1}, \ldots, Q_{r} \in Z_{2}$, we find a rational function $\mathfrak{f}$ on $\mathfrak{C}_{X_{0}, n}$ such that

$$
D_{0}=\sum_{j=1,2} \sum_{e^{\prime} \in E\left(v_{j}\right)} \operatorname{slp}_{e^{\prime}, v_{j}}\left(\mathfrak{f}_{\Gamma}\right) P_{e^{\prime}}^{v_{j}}
$$

and $\operatorname{div}(\mathfrak{f})+\mathcal{D}-\sum_{i} Q_{i} \geq 0$ and $\mathfrak{f}_{v} \in H_{v}$ for all $v$. Restricting to each $Z_{j}$, we have

$$
\mathcal{D}_{j}+D_{j}-\left(D_{j}-\left.D_{0}\right|_{Z_{j}}\right)+\operatorname{div}\left(\mathfrak{f}_{v_{j}}\right)-\sum_{k \in I_{j}} Q_{k} \geq 0
$$

for $j=1,2$, where $I_{1}=\{1, \ldots, r-l\}$ and $I_{2}=\{r-l+1, \ldots, r\}$. Moreover, we have

$$
\left.\left(\operatorname{div}\left(\mathfrak{f}_{\Gamma}\right)+\mathcal{D}_{\Gamma}\right)\right|_{e^{\circ}} \geq 0 \text {. }
$$

Since $\mathfrak{f}_{v_{j}} \in H_{j}$, it follows that

$$
\operatorname{dim}\left(H_{1} \cap H^{0}\left(Z_{1}, \mathscr{L}_{1}\left(-D_{1}+\left.D_{0}\right|_{Z_{1}}\right)\right)\right) \geq r-l+1
$$

and that

$$
\operatorname{dim}\left(H_{2} \cap H^{0}\left(Z_{2}, \mathscr{L}_{2}\left(-D_{2}+\left.D_{0}\right|_{Z_{2}}\right)\right)\right) \geq l+1 .
$$

Let $\lambda_{i}^{j}=-\operatorname{slp}_{e_{i}, v_{j}}\left(\mathfrak{f}_{\Gamma}\right)$. Since $H_{j} \subset H^{0}\left(Z_{j}, \mathscr{L}_{j}\right)$, we have

$$
\operatorname{dim}\left(H_{1} \cap H^{0}\left(Z_{1}, \mathscr{L}_{1}\left(-\left.D_{1}\right|_{\mathcal{A}_{1}}-\sum_{i} \lambda_{i}^{1} P_{i}^{1}\right)\right)\right) \geq r-l+1
$$

and that

$$
\operatorname{dim}\left(H_{2} \cap H^{0}\left(Z_{2}, \mathscr{L}_{2}\left(-\left.D_{2}\right|_{\mathcal{A}_{2}}-\sum_{i} \lambda_{i}^{2} P_{i}^{2}\right)\right)\right) \geq l+1 .
$$

We claim that there is an $a$ such that $\left.D_{1}\right|_{\mathcal{A}_{1}}+\sum \lambda_{i}^{1} P_{i}^{1} \geq D_{a}^{e, v_{1}}$ and $\left.D_{2}\right|_{\mathcal{A}_{2}}+\sum \lambda_{i}^{2} P_{i}^{2} \geq$ $D_{b-a}^{e, v_{2}}$, and hence $a_{l}^{e, v_{1}} \geq \operatorname{deg} D_{a}^{e, v_{1}}$ and $a_{r-l}^{e, v_{2}} \geq \operatorname{deg} D_{b-a}^{e, v_{2}}$, and the vanishing condition is satisfied.

Note that $\left.D_{1}\right|_{\mathcal{A}_{1}}=D_{\lambda}^{e, v_{1}}$ and $\left.D_{2}\right|_{\mathcal{A}_{2}}=D_{b-\lambda}^{e, v_{2}}$ for some $\lambda$. Let $n_{i}=\boldsymbol{n}\left(e_{i}\right)$, let $F_{i}:\left[0, n_{i}\right] \rightarrow \mathbb{R}$ be the piecewise linear function such that $F_{i}=\left.\mathfrak{f}_{\Gamma}\right|_{e_{i}}$. Without lost of generality assume $F_{i}(0)=0$ and $F_{i}\left(n_{i}\right)=y \geq 0$. We have $\lambda_{i}^{1}=-F_{i}^{\prime}(0)$ and $\lambda_{i}^{2}=F_{i}^{\prime}\left(n_{i}\right)$. Take $\widetilde{F}_{i}:\left[0, n_{i}\right] \rightarrow \mathbb{R}$ such that $\widetilde{F}_{i}(x)=0$ if $0 \leq x \leq x_{i}$ and $\widetilde{F}_{i}(x)=x-x_{i}$ otherwise. According to (3.1), $F_{i}(x)$ is convex when $x_{i}=0$ and $F_{i}(x)+\widetilde{F}_{i}(x)$ is 
convex when $x_{i} \neq 0$. We then have $\lambda_{i}^{1} \geq-\left\lfloor\frac{y}{n_{i}}\right\rfloor$ and $\lambda_{i}^{2} \geq\left\lceil\frac{y}{n_{i}}\right\rceil$ when $x_{i}=0$, and $\lambda_{i}^{1} \geq-\left\lfloor\frac{y+n_{i}-x_{i}}{n_{i}}\right\rfloor$ and $\lambda_{i}^{2} \geq\left\lceil\frac{y-x_{i}}{n_{i}}\right\rceil$ when $x_{i} \neq 0$.

Now it is enough to find $a$ such that

$$
\begin{aligned}
\sum_{i: x_{i}=0}\left\lfloor\frac{y}{n_{i}}\right\rfloor P_{i}^{1}+\sum_{i: x_{i} \neq 0}\left\lfloor\frac{y+n_{i}-x_{i}}{n_{i}}\right\rfloor P_{i}^{1} & \leq D_{\lambda}^{e, v_{1}}-D_{a}^{e, v_{1}} \\
& =\sum_{k=a}^{\lambda-1} \sum_{i: x_{i}-\lambda+k \equiv 0}\left(\bmod n_{i}\right)
\end{aligned}
$$

and

$$
\sum\left\lceil\frac{y-x_{i}}{n_{i}}\right\rceil P_{i}^{2} \geq D_{b-a}^{e, v_{2}}-D_{b-\lambda}^{e, v_{2}}=\sum_{k=b-\lambda}^{b-a-1} \sum_{i: x_{i}-\lambda+b-k \equiv 0} P_{\left(\bmod n_{i}\right)} P_{i}^{2}
$$

In other words we must have the following:

$$
\begin{aligned}
& \left\lfloor\frac{y}{n_{i}}\right\rfloor \leq \sum_{\substack{k: a \leq k \leq \lambda-1 \\
x_{i}-\lambda+k \equiv 0\left(\bmod n_{i}\right)}} 1, \\
& \left\lceil\frac{y}{n_{i}}\right\rceil \geq \sum_{\substack{k: b-\lambda \leq k \leq b-a-1 \\
x_{i}-\lambda+b-k=0}} 1 \text { if } x_{i}=0, \\
& \left\lfloor\frac{y+n_{i}-x_{i}}{n_{i}}\right\rfloor \leq \sum_{\substack{k: a \leq k \leq \lambda-1 \\
x_{i}-\lambda+k \equiv 0\left(\bmod n_{i}\right)}} 1, \\
& \left\lceil\frac{y-x_{i}}{n_{i}}\right\rceil \geq \sum_{\substack{k: b-\lambda \leq k \leq b-a-1 \\
x_{i}-\lambda+b-k \equiv 0}} 1 \text { if } x_{i} \neq 0 .
\end{aligned}
$$

It is straightforward to verify that $a=\lambda-\lfloor y\rfloor$ satisfies the inequalities above.

We next show that there is an isomorphism between

$$
P_{d}^{r}\left(X_{0}, \boldsymbol{n},\left(w_{v}\right)_{v}\right) \text { and } P_{d}^{r}\left(X_{0}, \boldsymbol{n},\left(w_{v}^{\text {red }}\right)_{v}\right)
$$

that is compatible with $\mathfrak{F}_{\left(w_{v}\right)_{v}}$ and $\mathfrak{F}_{\left(w_{v}^{\text {red }}\right)}$ for any tight tuple $\left(w_{v}\right)_{v}$. The bijectivity of $\mathfrak{F}_{\left(w_{v}\right)}$ then follows.

Corollary 3.10 For any tight tuple $\left(w_{v}\right)_{v}$ of admissible multidegrees in $V\left(G\left(w_{0}\right)\right)$, we have a natural isomorphism $\mathfrak{I}: P_{d}^{r}\left(X_{0}, \boldsymbol{n},\left(w_{v}\right)_{v}\right) \rightarrow P_{d}^{r}\left(X_{0}, \boldsymbol{n},\left(w_{v}^{\text {red }}\right)_{v}\right)$ such that $\mathfrak{F}_{\left(w_{v}\right)_{v}}=\mathfrak{F}_{\left(w_{v}^{\text {red }}\right)_{v}} \circ \mathfrak{I}$. Moreover, $\mathfrak{I}$ induces a bijection between the sets of pre-limit linear series that satisfy the weak glueing condition.

Proof Since $\mathfrak{F}_{\left(w_{v}^{\text {red }}\right) v}$ is a bijection, we define $\mathfrak{I}=\mathfrak{F}_{\left(w_{v}^{\text {red }}\right) v}^{-1} \circ \mathfrak{F}_{w_{0},\left(w_{v}\right)_{v}}$. To show that this is an isomorphism, we construct an inverse $\mathfrak{L}$ of $\mathfrak{I}$.

We first claim for all $v \in V(G)$ that $w_{v}$ is obtained from $w_{v}^{\text {red }}$ by twisting vertices in $V(G) \backslash\{v\}$. Suppose there is a path $P\left(w_{v}^{\text {red }}, v_{1}, \ldots, v_{m}\right)$ in $G\left(w_{0}\right)$ from $w_{v}^{\text {red }}$ to $w_{v}$ such that $\left\{v_{i}\right\}_{1 \leq i \leq m} \mp V(G)$. Take an ordering $v=v_{0}^{\prime}, v_{1}^{\prime}, v_{2}^{\prime}, \ldots$ of $V(G)$ such that $w_{v}$ becomes negative in $v_{i}^{\prime}$ for $i \geq 1$ after taking the negative twists of $v_{0}^{\prime}, \ldots, v_{i-1}^{\prime}$. Let $j$ be the smallest number such that $v_{j}^{\prime} \notin\left\{v_{i}\right\}_{1 \leq i \leq m}$. Then negatively twisting $w_{v}$ at 
$v_{0}^{\prime}, \ldots, v_{j-1}^{\prime}$ is the same as twisting $w_{v}^{\text {red }}$ at vertices in $\left\{v_{i}\right\}_{1 \leq i \leq m} \backslash\left\{v_{0}^{\prime}, \ldots, v_{j-1}^{\prime}\right\}$ (perhaps with multiplicities) that cannot be negative at $v_{j}^{\prime}$ unless $j=0$; namely, $v_{j}=v$, since $w_{v}^{\text {red }}$ is nonnegative at all vertices except $v$. So $v \notin\left\{v_{i}\right\}_{1 \leq i \leq m}$.

It follows that $D_{v}=D_{w_{v}^{\text {red }}, w_{v}}^{v}$ is effective for all $v$. Let $\left(\mathscr{L}_{v}, V_{v}\right)_{v} \in P_{d}^{r}\left(X_{0}, n\right.$, $\left.\left(w_{v}^{\text {red }}\right)_{v}\right)$. We define $\mathfrak{L}\left(\left(\mathscr{L}_{v}, V_{v}\right)_{v}\right)=\left(\mathscr{L}_{v}^{\prime}, V_{v}^{\prime}\right)_{v}$ where $\mathscr{L}_{v}^{\prime}=\mathscr{L}_{v}\left(D_{v}\right)$ and $V_{v}^{\prime}$ is the image of $V_{v}$ under the imbedding $i_{v}: H^{0}\left(Z_{v}, \mathscr{L}_{v}\right) \rightarrow H^{0}\left(Z_{v}, \mathscr{L}_{v}^{\prime}\right)$. We next show that $\left(\mathscr{L}_{v}^{\prime}, V_{v}^{\prime}\right)_{v}$ is contained in $P_{d}^{r}\left(X_{0}, \boldsymbol{n},\left(w_{v}\right)_{v}\right)$ and satisfies the weak glueing condition if and only if $\left(\mathscr{L}_{v}, V_{v}\right)_{v}$ does.

Take $e \in \bar{G}$ incident to $v$ and $v^{\prime}$. Suppose $w_{v}^{\text {red }}$ (resp., $w_{v}$ ) is obtained from $w_{v^{\prime}}^{\text {red }}$ (resp., $w_{v^{\prime}}$ ) by twisting $b_{v, v^{\prime}}$ (resp., $b_{v, v^{\prime}}^{\prime}$ ) times at $\left(e, v^{\prime}\right)$. Let $D_{i}^{e, v}$ (resp., $\left.\left(D_{i}^{e, v}\right)^{\prime}\right)$ be the twisting divisors associated with $\left(w_{v}^{\text {red }}\right)_{v}\left(\operatorname{resp} .,\left(w_{v}\right)_{v}\right)$. Let $\left(a_{l}^{e, v}\right)_{l}$ (resp., $\left.\left(\left(a_{l}^{e, v}\right)^{\prime}\right)_{l}\right)$ be the multivanishing sequence of $V_{v}$ (resp., $\left.V_{v}^{\prime}\right)$ along $D_{\bullet}^{e, v}$ (resp., $\left.\left(D_{\bullet}^{e, v}\right)^{\prime}\right)$.

Let $v_{1}, \ldots, v_{m}$ be as above. Also, take a path $P\left(w_{v^{\prime}}^{\text {red }}, v_{1}^{\prime}, \ldots, v_{m^{\prime}}^{\prime}\right)$ in $G\left(w_{0}\right)$ from $w_{v^{\prime}}^{\text {red }}$ to $w_{v^{\prime}}$ such that $v^{\prime} \notin\left\{v_{i}^{\prime}\right\}_{1 \leq i \leq m^{\prime}}$. Suppose $v$ appears $a$ times in $v_{1}^{\prime}, \ldots, v_{m^{\prime}}^{\prime}$ and $v^{\prime}$ appears $a^{\prime}$ times in $v_{1}, \ldots, v_{m}$. One easily verifies that $b_{v_{1}, v_{2}}^{\prime}=b_{v_{1}, v_{2}}+a^{\prime}+a$; and that if $a_{l}^{e, v}=\operatorname{deg} D_{j}^{e, v}$ for some $j$ critical, then $\left(a_{l}^{e, v}\right)^{\prime}=\operatorname{deg}\left(\left(D_{j+a^{\prime}}^{e, v}\right)^{\prime}\right)$ with $j+a^{\prime}$ critical; and if $a_{l}^{e, v^{\prime}}=\operatorname{deg} D_{j}^{e, v^{\prime}}$ for some $j$ critical, then $\left(a_{l}^{e, v^{\prime}}\right)^{\prime}=\operatorname{deg}\left(\left(D_{j+a}^{e, v^{\prime}}\right)^{\prime}\right)$ with $j+a$ critical. It then follows by definition that $\left(\mathscr{L}_{v}^{\prime}, V_{v}^{\prime}\right)_{v}$ is in $P_{d}^{r}\left(X_{0}, n,\left(w_{v}\right)_{v}\right)$.

Moreover, for $s \in V_{v}$ and $s^{\prime}=i_{v}(s) \in V_{v}^{\prime}$, we have $\operatorname{ord}_{P}^{0}(s)-\operatorname{ord}_{P}\left(D_{\bullet}^{e, v}\right)=$ $\operatorname{ord}_{P}^{0}\left(s^{\prime}\right)-\operatorname{ord}_{P}\left(\left(D_{\bullet+a^{\prime}}^{e, v}\right)^{\prime}\right)$ for $P \in \mathcal{A}_{e}^{v}$, and the same holds if we replace $v$ by $v^{\prime}$ and $a^{\prime}$ by $a$. In particular, if $W_{v, j} \subset V_{v}\left(-D_{j}^{e, v}\right) / V_{v}\left(-D_{j+1}^{e, v}\right)$ and

$$
W_{v^{\prime}, b_{v, v^{\prime}}-j} \subset V_{v^{\prime}}\left(-D_{b_{v, v^{\prime}}-j}^{e, v^{\prime}}\right) / V_{v^{\prime}}\left(-D_{b_{v, v^{\prime}}-j+1}^{e, v^{\prime}}\right)
$$

as in Definition 3.3 satisfies the weak glueing condition for $\left(w_{v}^{\text {red }}\right)_{v}$, then we can take $W_{v, j+a^{\prime}}^{\prime}=i_{v}\left(W_{v, j}\right) \subset V_{v}^{\prime}$ and $W_{v^{\prime}, b_{v, v^{\prime}}-j+a}^{\prime}=i_{v^{\prime}}\left(W_{v^{\prime}, b_{v, v^{\prime}}-j}\right) \subset V_{v^{\prime}}^{\prime}$ which fulfills the weak glueing condition for $\left(w_{v}\right)_{v}$, and vice versa.

Corollary 3.11 For any tight tuple $\left(w_{v}\right)_{v}$ in $V\left(G\left(w_{0}\right)\right)$ the map

$$
\mathfrak{F}_{\left(w_{v}\right)_{v}}: P_{d}^{r}\left(X_{0}, n,\left(w_{v}\right)_{v}\right) \longrightarrow \mathfrak{G}_{d}^{r}\left(\mathfrak{C}_{X_{0}, n}, w_{0}\right)
$$

is a bijection, the preimage of $(\mathcal{D}, \mathcal{H})$ with $\mathcal{D}_{\Gamma}=D_{w_{0}}$ is $\left(\mathscr{O}_{Z_{v}}\left(\mathcal{D}_{v}+D_{w_{0}, w_{v}}^{v}\right), H_{v}\right)_{v}$. In particular, we have $H_{v} \subset H^{0}\left(Z_{v}, \mathscr{O}_{Z_{v}}\left(\mathcal{D}_{v}+D_{w_{0}, w_{v}}^{v}\right)\right)$.

We are now able to define the weak glueing condition for limit linear series on $\mathfrak{C}_{X_{0}, n}$ of multidegree $w_{0}$ by lifting to a pre-limit linear series on $\left(X_{0}, n\right)$, and the definition is independent of the choice of lifting by Corollary 3.10 .

Definition 3.12 Let $(\mathcal{D}, \mathcal{H}) \in \mathfrak{G}_{d}^{r}\left(\mathfrak{C}_{X_{0}, n}, w_{0}\right)$ be a limit $\mathfrak{g}_{d}^{r}$ on $\mathfrak{C}_{X_{0}, n}$. We say that $(\mathcal{D}, \mathcal{H})$ satisfies the weak glueing condition with respect to $w_{0}$ if $\mathfrak{F}_{\left(w_{v}\right)_{v}}^{-1}((\mathcal{D}, \mathcal{H}))$ satisfies the weak glueing condition with respect to $\left(w_{v}\right)_{v}$ for a tight tuple $\left(w_{v}\right)_{v}$ in $V\left(G\left(w_{0}\right)\right)$. 
Proposition $3.13 \quad$ (i) Suppose $(\mathcal{D}, \mathcal{H}) \in \mathfrak{G}_{d}^{r}\left(\mathfrak{C}_{X_{0}, n}, w\right)$ for $w=w_{0}$ and $w=w_{0}^{\prime}$. Then $(\mathcal{D}, \mathcal{H})$ satisfies the weak glueing condition with respect to $w_{0}$ if and only if it satisfies that with respect to $w_{0}^{\prime}$.

(ii) Suppose $w_{0}=\left(w_{G}, \mu\right)$. Take $m \in \mathbb{Z}_{\geq 0}$ and $\boldsymbol{n}^{\prime}: E(G) \rightarrow \mathbb{Z}_{>0}$ such that $\boldsymbol{n}^{\prime}(e)=$ $m n(e)$ for all $e \in E(G)$. Let $\left(\mathcal{D}^{\prime}, \mathcal{H}^{\prime}\right)$ be a limit $\mathfrak{g}_{d}^{r}$ on $\mathfrak{C}_{X_{0}, n^{\prime}}$ such that $H_{v}^{\prime}=H_{v}$ and $\mathcal{D}^{\prime}$ corresponds to $w^{\prime}=\left(w_{G}^{\prime}, \mu^{\prime}\right)$ where $w_{G}^{\prime}=w_{G}$ and $\mu^{\prime}(e)=m \mu(e)$ for all $e \in E(G)$. Then $(\mathcal{D}, \mathcal{H})$ satisfies the weak glueing condition if and only if $\left(\mathcal{D}^{\prime}, \mathcal{H}^{\prime}\right)$ satisfies the weak glueing condition.

Proof (i) Note that Definition 3.12 only depends on $V\left(G\left(w_{0}\right)\right)$. We have $D_{w_{0}}$ linearly equivalent to $D_{w_{0}^{\prime}}$ as divisors on $\Gamma$. It is then easy to check that $w_{0} \in V\left(G\left(w_{0}^{\prime}\right)\right)$ and hence $G\left(w_{0}\right)=G\left(w_{0}^{\prime}\right)$.

(ii) This is obvious; note that the scaling of a concentrated admissible multidegree is still concentrated.

Now we define the weak glueing condition for arbitrary limit linear series on $\mathfrak{C}_{X_{0}, n}$.

Definition 3.14 We say that a limit linear series $(\mathcal{D}, \mathcal{H})$ on $\mathfrak{C}_{X_{0}, n}$ satisfies the weak glueing condition if after scaling (by integer) of $\boldsymbol{n}$ there is a multidegree $w_{0}$ on $(G, \boldsymbol{n})$ such that $(\mathcal{D}, \mathcal{H})$ is contained in $\mathfrak{G}_{d}^{r}\left(\mathfrak{C}_{X_{0}, n}, w_{0}\right)$ and satisfies the weak glueing condition with respect to $w_{0}$.

Note that the definition above is independent of the choice of $w_{0}$ and scaling by Proposition 3.13. See Example 4.11 for a limit linear series on a metrized complex that does not satisfy the weak glueing condition.

\section{Smoothing of Limit Linear Series on Metrized Complexes}

In this section we use the same symbols as in Notation 3.1 and assume that $\kappa$ is algebraically closed. We consider special cases of $w_{0}$ and $\left(X_{0}, n\right)$ such that (1) the space of limit linear series on $\left(X_{0}, n\right)$ is of expected dimension, and (2) any pre-limit linear series satisfying the weak glueing condition lifts to a limit linear series on $\left(X_{0}, n\right)$. This along with the forgetful map constructed in the last section will guarantee the smoothability of certain limit linear series on $\mathfrak{C}_{X_{0}, n}$.

In order to prove the main theorems, we first recall some results from [Oss] and [Oss16] for convenience.

Theorem 4.1 ([Oss, Theorem 6.1]) If $\pi: X \rightarrow \operatorname{Spec}(R)$ is a regular smoothing family with special fiber $\widetilde{X}_{0}$, and the space of limit linear series on $\left(X_{0}, n\right)$ has the expected dimension $\rho=g+(r+1)(d-r-g)$, then every limit linear series on $\left(X_{0}, n\right)$ can be smoothed to a linear series on the generic fiber $X_{\eta}$.

Definition 4.2 Let $X$ be a smooth projective curve of genus $g$, and fix integers $r, d, n>0$, and for $i=1, \ldots, n$ fix also $m_{i}>0$. Choose distinct points $P_{i}^{k}$ on $X$ for $i=1, \ldots, n$ and $k=1, \ldots, m_{i}$. Then we say that $\left(X,\left(P_{i}^{k}\right)_{i, k}\right)$ is strongly BrillNoether general for $r, d$ if, for all tuples of nondecreasing effective divisor sequences $D_{\bullet}^{i}$, such that every divisor $D_{\bullet}^{i}$ is supported on $P_{i}^{1}, \ldots, P_{i}^{m_{i}}$, and for every tuple of 
nondecreasing sequences $a_{\bullet}^{i}$ such that for each $j$ we have $a_{j}^{i}=\operatorname{deg} D_{l}^{i}$ for some $\ell$ critical for $D_{\bullet}^{i}$ and the number of repetitions of $a_{j}^{i}$ is at most $\operatorname{deg}\left(D_{\ell+1}^{i}-D_{\ell}^{i}\right)$, the space $G_{d}^{r}\left(X,\left(D_{\bullet}^{i}, a_{\bullet}^{i}\right)_{i}\right)$ of $\mathfrak{g}_{d}^{r}$ s on $X$ having multivanishing sequence at least $a_{\bullet}^{i}$ along

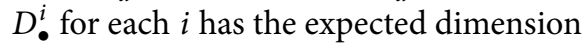

$$
\rho:=g+(r+1)(d-r-g)-\sum_{i=1}^{n}\left(\sum_{j=0}^{r}\left(a_{j}^{i}-j\right)+\sum_{\ell=0}^{b_{i}}\left(\begin{array}{c}
r_{\ell}^{i} \\
2
\end{array}\right)\right)
$$

if it is nonempty. In the above, $D_{\bullet}^{i}$ is indexed from 0 to $b_{i}+1$, and $r_{\ell}^{i}$ is defined to be 0 if $\ell$ is not critical for $D_{\bullet}^{i}$, and the number of times $\operatorname{deg} D_{\ell}^{i}$ occurs in $a_{\bullet}^{i}$ if $\ell$ is critical.

See [Oss16, Theorem 3.3] for examples of strongly Brill-Noether general curves. In particular, a rational curve is strongly Brill-Noether general for $n \leq 2$. This example will be used in Section 5 .

Theorem 4.3 ([Oss16, Corollary 5.2]) Suppose $\left(X_{0}, n\right)$ satisfies the following conditions:

(i) there are at most three edges of $G$ connecting any given pair of vertices;

(ii) there exists a $d^{\prime}$ such that for any adjacent vertices $v, v^{\prime}$ of $G$, connected by edges $\left(e_{i}\right)_{i}$, and any integers $\left(x_{i}\right)_{i}$ with $\sum_{i} x_{i} n\left(e_{i}\right)=0$, if there is a unique $j$ with $x_{j}>0$, then we have $\sum_{i}\left\lfloor x_{j} n\left(e_{j}\right) / n\left(e_{i}\right)\right\rfloor>d^{\prime}$;

(iii) each marked component of $X_{0}$ is strongly Brill-Noether general.

Then the space of limit linear series on $\left(X_{0}, n\right)$ (of multidegree $w_{0}$ ) of degree $d$ with $d \leq d^{\prime}$ is pure of expected dimension $\rho$.

We first let $\boldsymbol{n}$ vary. For a family of $w_{0}$, conditions (i) and (ii) in the beginning of this section are satisfied for all $X_{0}$ with strongly Brill-Noether general components. Moreover, the weak glueing condition is satisfied automatically.

Theorem 4.4 Let $\left\{e_{j}^{i}\right\}_{1 \leq i \leq k_{j}}$ be all edges of $G$ over an edge $e_{j} \in E(\bar{G})=\left\{e_{j}\right\}_{j \in J}$. Let $(\mathcal{D}, \mathcal{H})$ be a limit $\mathfrak{g}_{d}^{r}$ on $\mathfrak{C}_{X_{0}, n}$ with rational and edge-reduced $\mathcal{D}$. Suppose $G$ is directed such that the edges over the same edge of $\bar{G}$ have the same tail. Let $x_{j}^{i} \in\left[0, n\left(e_{j}^{i}\right)\right)$ correspond to the point in $\mathcal{D} \cap\left(e_{j}^{i}\right)^{\circ}$ where we set $x_{j}^{i}=0$ if the intersection is empty. Suppose that the following hold:

(i) There is a number $m \in \mathbb{Z}$ such that $m x_{j}^{i} \in \mathbb{Z}$ for all $j$ and $1 \leq i \leq k_{j}$, and for each $j$ the classes of $m x_{j}^{i}$ where $1 \leq i \leq k_{j} \operatorname{modulo} \operatorname{gcd}\left(m n\left(e_{j}^{1}\right), \ldots, m n\left(e_{j}^{k_{j}}\right)\right)$ are distinct. (ii) Each component of $X$ is strongly Brill-Noether general.

Then the limit linear series $(\mathcal{D}, \mathcal{H})$ is smoothable.

Note that if $\mathcal{D}$ is of multidegree $w_{0}=\left(w_{G}, \mu\right)$, then Theorem 4.4(i) says that, with the given direction of $G$ in Theorem 4.4, for each $j$ the classes of $\mu\left(e_{j}^{i}\right)$ where $1 \leq i \leq k_{j}$ $\operatorname{modulo} \operatorname{gcd}\left(n\left(e_{j}^{1}\right), \ldots, n\left(e_{j}^{k_{j}}\right)\right)$ are distinct.

Lemma 4.5 Let $\left(\mathscr{L},\left(V_{v}\right)_{v}\right)$ be a limit $\mathfrak{g}_{d}^{r}$ on $\left(X_{0}, n\right)$ of multidegree $w_{0}$ with respect to $\left(w_{v}\right)_{v}$, and let $\left(\mathscr{L}^{v}, V_{v}\right)_{v}$ be the induced pre-limit linear series. Let $X \rightarrow \operatorname{Spec}(R)$ be 
a regular smoothing family with special fiber $\widetilde{X}_{0}$. If $\left(\mathscr{L},\left(V_{v}\right)_{v}\right)$ can be smoothed to a $\mathfrak{g}_{d}^{r}$ on $X_{\eta}$, then $\mathfrak{F}\left(\left(\mathscr{L}^{v}, V_{v}\right)_{v}\right)$ is smoothable in Amini-Baker sense.

Proof This follows directly from [Oss17, Proposition 4.16].

Proof of Theorem 4.4 Up to scaling, we can assume that $\mathcal{D}$ is integral and of multidegree $w_{0}$, hence we can take $m=1$. Take a pre-limit linear series $\left(\mathscr{L}_{v}, V_{v}\right)_{v}$ with respect to $\left(w_{v}\right)_{v}$ that maps to $(\mathcal{D}, \mathcal{H})$ under $\mathfrak{F}_{\left(w_{v}\right)_{v}}$. Then condition (i) implies that $\operatorname{deg}\left(D_{j+1}^{e, v}\right)-\operatorname{deg}\left(D_{j}^{e, v}\right)=1$, and the morphism $\varphi_{j}^{(e, v)}$ in Definition 2.17 is a linear morphism between one dimensional spaces. Hence for any line bundle $\mathscr{L}$ on $\widetilde{X}_{0}$ of multidegree $w_{0}$ such that $\mathscr{L}^{v}=\mathscr{L}_{v}$ and any enriched structure on $\widetilde{X}_{0}$ the condition (ii) of Definition 2.17 is satisfied automatically. In other words $\left(\mathscr{L},\left(V_{v}\right)_{v}\right)$ is a limit linear series. By Lemma 4.5 , it remains to show that $\left(\mathscr{L},\left(V_{v}\right)_{v}\right)$ is smoothable. According to [Bak08, Theorem B.2], there is a regular smoothing family $X$ over $B$ with special fiber $\widetilde{X}_{0}$, and Theorem 4.1 says that $\left(\mathscr{L},\left(V_{v}\right)_{v}\right)$ can be smoothed to a $\mathfrak{g}_{d}^{r}$ on $X_{\eta}$ if the the space $G_{w_{0}}^{r}\left(X_{0}, n,\left(\mathscr{O}_{v}\right)_{v}\right)$ of limit $\mathfrak{g}_{d}^{r} \operatorname{son}\left(X_{0}, n\right)$ has dimension $\rho$, where $\left(\mathscr{O}_{v}\right)_{v}$ is the enriched structure induced by $X$. This is ensured by condition (ii) and [Oss16, Theorem 4.1], since there is no glueing condition imposed.

Theorem 4.4(i) is trivial when $X_{0}$ is of compact type, whereas condition (ii) is still necessary; see [AB15, Example 5.14] for a counterexample. As a result we have the following corollary.

Corollary 4.6 Let $X_{0}$ be a curve of compact type, and assume the marked components of $X_{0}$ are all strongly Brill-Noether general. Let $(\mathcal{D}, \mathcal{H})$ be a limit linear series on the metrized complex $\mathfrak{C}_{X_{0}, n}$ with rational $\mathcal{D}$. Then $(\mathcal{D}, \mathcal{H})$ is smoothable.

We next consider $\left(X_{0}, n\right)$ as in Theorem 4.3 and prove that the weak glueing condition is a necessary and sufficient condition for the smoothability of a limit linear series on $\mathfrak{C}_{X_{0}, n}$ (of certain degree). The necessity is actually valid for arbitrary $\left(X_{0}, n\right)$.

Theorem 4.7 Suppose $(\mathcal{D}, \mathcal{H})$ is a smoothable limit $\mathfrak{g}_{d}^{r}$ on $\mathfrak{C}_{X_{0}, n}$. Then $(\mathcal{D}, \mathcal{H})$ satisfies the weak glueing condition.

Note that in the following proof we do not assert that $(\mathcal{D}, \mathcal{H})$ is the image (under $\left.\mathfrak{F}_{\left.\left(w_{v}\right)_{v}\right)}\right)$ of a smoothable limit linear series on $\left(X_{0}, \boldsymbol{n}\right)$, as $\mathfrak{F}\left(\left(\mathscr{L}^{v}, V_{v}\right)_{v}\right)$ in Lemma 4.5. It is unclear whether a strongly semistable model (when the associated metrized complex has identical edge lengths) over $\widetilde{R}$ is a base extension of a regular smoothing family over $R$, and we do not know if the specialization of any $\mathfrak{g}_{d}^{r}$ on the generic fiber of a strongly semistable model can be lifted to the specialization of a $\mathfrak{g}_{d}^{r}$ on the generic fiber of a regular smoothing family.

Proof of Theorem 4.7 Since $\mathcal{D}_{\Gamma}$ is linearly equivalent to a rational divisor on $\Gamma$, we can assume that $\mathcal{D}$ is integral and edge-reduced and $\mathcal{D}_{\Gamma}=D_{w_{0}}$. Suppose there is a strongly semistable model $\mathfrak{X}$ over $\widetilde{R}$ with associated metrized graph $\mathfrak{C X}=\mathfrak{C}_{X_{0}, n}$ and a linear series $\left(D_{\eta}, H_{\eta}\right)$ on $\mathfrak{X}_{\eta}$ of rank $r$ that specializes to $(\mathcal{D}, \mathcal{H})$. 
Suppose the concentrated degrees $\left(w_{v}\right)_{v}=\left(w_{v}^{\text {red }}\right)_{v}$ are given. Let $D_{v}=D_{w_{0}, w_{v}^{\text {red }}}^{v}$. We have $H_{v} \subset H^{0}\left(Z_{v}, \mathscr{O}_{Z_{v}}\left(\mathcal{D}_{v}+D_{v}\right)\right)$. Consider $v$ and $v^{\prime}$ in $V(\bar{G})$ connected by $e \in E(\bar{G})$. Let $\left\{e_{i}\right\} \subset E(G)$ be the edges lying over $e$, let $P_{i}=P_{e_{i}}^{v}$ and $P_{i}^{\prime}=P_{e_{i}}^{v^{\prime}}$. Take $h \in H_{v} \subset H^{0}\left(Z_{v}, \mathscr{O}_{Z_{v}}\left(\mathcal{D}_{v}+D_{v}\right)\right)$. Choose $g \in H_{\eta}$ such that $\tau_{* v}^{\mathfrak{C X}}(g)=h$ up to multiplication by $\kappa^{\times}$. Let $h^{\prime}=\tau_{* v^{\prime}}^{\mathfrak{C X}}(g)$. According to the Slope Formula [AB15, Theorem 4.4(3)], we have

$$
\operatorname{ord}_{P_{i}}(h)=-\operatorname{slp}_{e_{i}, v}\left(\tau_{*}^{\mathfrak{C X}}(g)_{\Gamma}\right) \quad \text { and } \quad \operatorname{ord}_{P_{i}^{\prime}}\left(h^{\prime}\right)=-\operatorname{slp}_{e_{i}, v^{\prime}}\left(\tau_{*}^{\mathfrak{C X}}(g)_{\Gamma}\right) .
$$

On the other hand, since $D_{\eta}+\operatorname{div}(g) \geq 0$, by [AB15, Theorem 4.5] we have

$$
0 \leq \tau_{*}^{\mathfrak{C X}}\left(D_{\eta}+\operatorname{div}(g)\right)=\mathcal{D}+\operatorname{div}\left(\tau_{*}^{\mathfrak{C X}}(g)\right) .
$$

Restricting to $e_{i}^{\circ}$, where $e_{i}$ is considered as an edge of $\Gamma$ (with vertex set $V(G)$ ), we have

$$
\left.\mathcal{D}\right|_{e_{i}^{\circ}}+\left.\operatorname{div}\left(\tau_{*}^{\mathfrak{C X}}(g)_{\Gamma}\right)\right|_{e_{i}^{\circ}} \geq 0 .
$$

By (4.1), for each $j$ we have (note that $\mathcal{D}_{v}$ is supported on $Z_{v} \backslash \mathcal{A}_{v}$ )

$$
\operatorname{ord}_{P_{i}}^{0}(h)-\operatorname{ord}_{P_{i}}\left(D_{j}^{e, v}\right)=\operatorname{slp}_{e_{i}, v}\left(-\tau_{*}^{\mathfrak{C X}}(g)_{\Gamma}\right)-\operatorname{ord}_{P_{i}}\left(D_{j}^{e, v}-D_{v}\right) \text {. }
$$

Let $w_{v}^{j}$ be the multidegree obtained from $w_{v}$ by twisting $j$ times at $(e, v)$ and $\lambda$ be the piecewise linear function on $\Gamma$ such that $\operatorname{div}(\lambda)=\mathcal{D}_{\Gamma}-D_{w_{v}^{j}}$. Let $F_{g}=-\tau_{*}^{\mathfrak{C X}}(g)_{\Gamma}-\lambda$. We then have $\operatorname{ord}_{P_{i}}\left(D_{j}^{e, v}-D_{v}\right)=\operatorname{slp}_{e_{i}, v}(\lambda)$ and

$$
\operatorname{ord}_{P_{i}}^{0}(h)-\operatorname{ord}_{P_{i}}\left(D_{j}^{e, v}\right)=\operatorname{slp}_{e_{i}, v}\left(F_{g}\right) .
$$

A symmetric argument shows that

$$
\operatorname{ord}_{P_{i}^{\prime}}^{0}\left(h^{\prime}\right)-\operatorname{ord}_{P_{i}^{\prime}}\left(D_{r-j}^{e, v^{\prime}}\right)=\operatorname{slp}_{e_{i}, v^{\prime}}\left(F_{g}\right) \text {. }
$$

According to (4.2) we have

$$
\left.\operatorname{div}\left(-F_{g}\right)\right|_{e_{i}^{\circ}}=\left.\mathcal{D}\right|_{e_{i}^{\circ}}+\left.\operatorname{div}\left(\tau_{*}^{\mathfrak{C X}}(g)_{\Gamma}\right)\right|_{e_{i}^{\circ}}+\left.(\operatorname{div}(\lambda)-\mathcal{D})\right|_{e_{i}^{\circ}} \geq-\left.D_{w_{v}^{j}}\right|_{e_{i}^{\circ}} .
$$

Thus, the same argument as in the proof of Theorem 3.9 shows that there is an $a$ such that

$$
\operatorname{ord}_{D_{\bullet}^{e, v}}(h) \geq \operatorname{deg} D_{a}^{e, v^{\prime}} \quad \text { and } \quad \operatorname{ord}_{D_{\bullet}^{e, v^{\prime}}}\left(h^{\prime}\right) \geq \operatorname{deg} D_{b_{v, v^{\prime}}, a}^{e, v^{\prime}} .
$$

Now let $h_{0}, \ldots, h_{r}$ be a basis of $H_{v}$ such that $\operatorname{ord}_{D_{\bullet}^{e, v}}\left(h_{i}\right)=a_{i}^{e, v}$, where $\left\{a_{i}^{e, v}\right\}$ is the multivanishing sequence of $\left(\mathscr{O}_{Z_{v}}\left(\mathcal{D}_{v}+D_{v}\right), H_{v}\right)$ along $D_{\bullet}^{e, v}$. Let $j$ be a critical number for $D_{\bullet}^{e, v}$. It follows that $b_{v, v^{\prime}}-j$ is critical for $D_{\bullet}^{e, v^{\prime}}$. Assume $H_{v}\left(-D_{j+1}^{e, v}\right)$ is generated by $\left\{h_{l+1}, \ldots, h_{r}\right\}$. Then $\operatorname{ord}_{D_{\bullet}^{e, v}}(\ell) \leq \operatorname{deg}\left(D_{j}^{e, v}\right)$ for $\ell \in \operatorname{span}\left(h_{0}, \ldots, h_{l}\right)$. Let $g_{0}, \ldots, g_{l}$ be rational functions in $H_{\eta}$ such that $\tau_{* v}^{\mathfrak{C X}}\left(g_{i}\right)=h_{i}$. Then $L=\operatorname{span}\left(g_{0}, \ldots, g_{l}\right)$ is an $(l+1)$-dimensional subspace of $H_{\eta}$ such that $\tau_{* v}^{\mathfrak{C X}}(L)=\operatorname{span}\left(h_{0}, \ldots, h_{l}\right)$ and $\tau_{* v^{\prime}}^{\mathfrak{C X}}(L)$ is an $(l+1)$-dimensional subspace of $H_{v^{\prime}}$.

It follows from (4.6) that for any $g \in L$ we have $\tau_{* v^{\prime}}^{\mathfrak{C X}}(g) \in H_{v^{\prime}}\left(-D_{b_{v, v^{\prime}}-j}^{e, v^{\prime}}\right)$. Now we can pick the spaces $W_{v, j}$ and $W_{v^{\prime}, b_{v, v^{\prime}}-j}$ (of dimension $g_{j}$ ) as described in Definition 3.3. Note that $g_{j} \leq l+1-\operatorname{dim}\left(H_{v^{\prime}}\left(-D_{b_{v, v^{\prime}}-j+1}^{e, v^{\prime}}\right)\right)$. Taking $W_{v^{\prime}, b_{v, v^{\prime}}-j}$ to be an arbitrary subspace of $\tau_{* v^{\prime}}^{\mathfrak{C X}}(L)$ of dimension $g_{j}$ such that $W_{v^{\prime}, b_{v, v^{\prime}}-j} \cap H_{v^{\prime}}\left(-D_{b_{v, v^{\prime}}}^{e, v^{\prime}}\right)=0$ and 
$W_{v, j}=\tau_{* v}^{\mathfrak{C X}}(W)$, where $W$ is a $g_{j}$ dimensional subspace of $L$ such that $\tau_{* v^{\prime}}^{\mathfrak{C X}}(W)=$ $W_{v^{\prime}, b_{v, v^{\prime}}-j}$ (which can be constructed in the same way as $L$ ), will complete the proof. Indeed, for all $s^{\prime} \in W_{v^{\prime}, b_{v, v^{\prime}}-j}$, taking $t \in W$ such that $\tau_{* v^{\prime}}^{\mathfrak{C X}}(t)=s^{\prime}$ and $s=\tau_{* v}^{\mathfrak{C X}}(t) \in$ $\operatorname{span}\left(h_{0}, \ldots, h_{l}\right)$ we have that $\operatorname{ord}_{D_{\bullet}^{e, v}}\left(s^{\prime}\right)=D_{b_{v, v^{\prime}}-j}^{e, v^{\prime}}$, and hence $\operatorname{ord}_{D_{\bullet}^{e, v}}(s)=D_{j}^{e, v}$ by (4.6). On the other hand, we have $-\left.D_{w_{v}^{j}}\right|_{e_{i}^{\circ}}=\varnothing$ and hence $F_{g}$ is a concave function on $e_{i}^{\circ}$ by (4.5) for all $i$ such that $P_{i}$ is in the support of $D_{j+1}-D_{j}$. Therefore for the same set of $i$ we have that $s$ vanishes at $P_{i}$ if and only if $s^{\prime}$ vanishes at $P_{i}^{\prime}$ by (4.3) and (4.4) where $s$ (resp., $\left.s^{\prime}\right)$ is considered in $H_{v}\left(-D_{j}^{e, v}\right) / H_{v}\left(-D_{j+1}^{e, v}\right)$ (resp., $\left.H_{v^{\prime}}\left(-D_{b_{v, v^{\prime}}-j}^{e, v^{\prime}}\right) / H_{v^{\prime}}\left(-D_{b_{v, v^{\prime}}-j+1}^{e, v^{\prime}}\right)\right)$. Now the conclusion follows from Remark 3.4.

We now focus on the case of Theorem 4.3.

Theorem 4.8 Let $\left(X_{0}, n\right)$ and $d^{\prime}$ be as in Theorem 4.3. Then for any limit linear series $(\mathcal{D}, \mathcal{H})$ on $\mathfrak{C}_{X_{0}, n}$ of multidegree $w_{0}$ with $\operatorname{deg}(\mathcal{D}) \leq d^{\prime}$ the following are equivalent.

(i) $(\mathcal{D}, \mathcal{H})$ is smoothable;

(ii) $(\mathcal{D}, \mathcal{H})$ satisfies the weak glueing condition;

(iii) $(\mathcal{D}, \mathcal{H})$ is the image under $\mathfrak{F}_{\left(w_{v}\right)}$, of a pre-limit linear series on $\left(X_{0}, n\right)$ that lifts to a limit linear series.

Moreover we have (i) $\Leftrightarrow$ (ii) when $\mathcal{D}$ is only required to be rational.

Lemma 4.9 Let $\left(X_{0}, n\right)$ be as in Theorem 4.8 and $d \leq d^{\prime}$. Then a pre-limit linear series $\left(\mathscr{L}_{v}, V_{v}\right)_{v}$ with respect to $\left(w_{v}\right)_{v}$ lifts to a limit linear series if and only if $\left(\mathscr{L}_{v}, V_{v}\right)_{v}$ satisfies the weak glueing condition.

Proof By Proposition 3.6 it remains to prove the "if" part. Take any line bundle $\mathscr{L}$ on $\widetilde{X}_{0}$ of multidegree $w_{0}$ such that $\mathscr{L}^{v}=\mathscr{L}_{v}$. For any adjacent vertices $v, v^{\prime} \in V(G)$ connected by $e \in E(\bar{G})$, according to the proof of Theorem 4.3 we have one of the following:

(a) there is at most one $j$ such that $\operatorname{deg} D_{j+1}^{e, v}-\operatorname{deg} D_{j}^{e, v}=3$ while $\operatorname{deg} D_{j^{\prime}+1}^{e, v}-$ $\operatorname{deg} D_{j^{\prime}}^{e, v} \leq 1$ for $j^{\prime} \neq j$ and $0 \leq j^{\prime} \leq b_{v, v^{\prime}}$;

(b) there are at most two numbers $\left\{j_{i}\right\}_{1 \leq i \leq m}$ where $0 \leq j_{i} \leq b_{v, v^{\prime}}$ and $m \leq 2$ such that $\operatorname{deg} D_{j_{i}}^{e, v}-\operatorname{deg} D_{j_{i}-1}^{e, v}=2$, and when $m=2$ the supports of $D_{j_{1}+1}^{e, v}-D_{j_{1}}^{e, v}$ and $D_{j_{2}+1}^{e, v}-D_{j_{2}}^{e, v}$ only overlap at one point.

For any given enriched structure $\left(\mathscr{O}_{v}\right)_{v}$ we check that Definition 2.17(ii) will be satisfied after adjusting the glueing data of $\mathscr{L}$ along the edges lying over $e$, which has dimension $c=\#\left\{e^{\prime} \mid e^{\prime} \in E(G)\right.$ lies over $\left.e\right\}-1$. The nontrivial cases are when $\operatorname{deg} D_{j+1}^{e, v}-\operatorname{deg} D_{j}^{e, v}=3$ and $g_{j} \leq 2$, which impose codimension at most 2 (when $g_{j}=2$ we can take basis of $V\left(-D_{j}^{e, v}\right) / V\left(-D_{j+1}^{e, v}\right)$ that vanish at different points, each of which impose codimension at most one); or when $\operatorname{deg} D_{j+1}^{e, v}-\operatorname{deg} D_{j}^{e, v}=2$ and $g_{j}=1$, which impose codimension at most 1 . Now the first case happens only if $c=2$ and the second case happens when $m=1$ and $c \geq 1$ or $m=2$ and $c=2$. 
Proof of Theorem 4.8 We have (i) $\Rightarrow$ (ii) according to Theorem 4.7. Lemma 4.9, Theorem 4.3, and the smoothing theorem (Theorem 4.1) together show (ii) $\Rightarrow$ (iii). And (iii) $\Rightarrow$ (i) is Lemma 4.5 .

When $\rho=0$, the weak glueing condition in Theorem 4.8 is trivial, since according to the proof of Theorem 4.3 , the space of pre-limit $\mathfrak{g}_{d}^{r} s$ with extra vanishing has dimension strictly less that $\rho$, which is empty. As a result we have the following corollary.

Corollary 4.10 Let $\left(X_{0}, n\right)$ be as in Theorem 4.8 , and $(\mathcal{D}, \mathcal{H})$ a limit $\mathfrak{g}_{d}^{r}$ of multidegree $w_{0}$ such that $d \leq d^{\prime}$ and $\rho=0$. Then $(\mathcal{D}, \mathcal{H})$ is smoothable.

Example 4.11 We give an example of a limit linear series on a metrized complex that does not satisfy the weak glueing condition, hence is not smoothable. Let $X_{0}$ be a nodal curve obtained by glueing two copies of $\mathbb{P}_{\kappa}^{1}$ along two points. Let $V(G)=$ $\left\{v, v^{\prime}\right\}$ and $E(G)=\left\{e_{1}, e_{2}\right\}$. Let $n$ be the chain structure such that $n\left(e_{1}\right)=2$ and $n\left(e_{2}\right)=1$. Let $\mathcal{A}_{v}=\{P, Q\}$ and $A_{v^{\prime}}=\left\{P^{\prime}, Q^{\prime}\right\}$. The metrized complex is as below, where $\widetilde{P}$ is the midpoint of $e_{1}$.

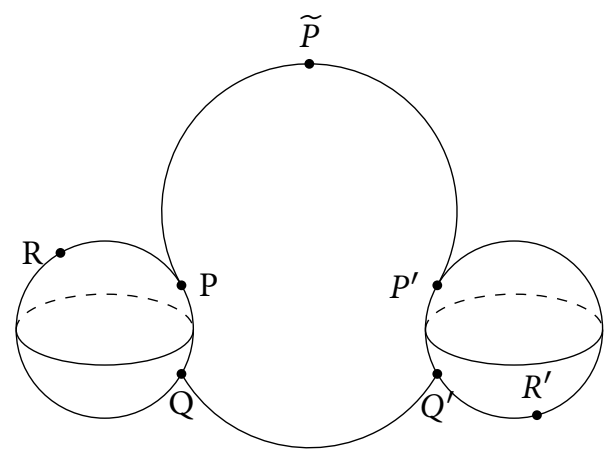

Consider $(\mathcal{D}, \mathcal{H})$ such that $\mathcal{D}_{v}=2 R, \mathcal{D}_{\Gamma}=2 v, \mathcal{D}_{v^{\prime}}=0, H_{v}=\left\langle f_{0}, f_{1}\right\rangle$, and $H_{v^{\prime}}=$ $\left\langle f_{0}^{\prime}, f_{1}^{\prime}\right\rangle$, where $\operatorname{div}\left(f_{0}\right)=P-R, \operatorname{div}\left(f_{1}\right)=P+Q-2 R, \operatorname{div}\left(f_{0}^{\prime}\right)=R^{\prime}-Q^{\prime}$, and $\operatorname{div}\left(f_{1}^{\prime}\right)=0$ (namely $f_{1}^{\prime}$ is a nonzero constant function), for some $R \in Z_{v} \backslash\{P, Q\}$ and $R^{\prime} \in Z_{v^{\prime}} \backslash\left\{P^{\prime}, Q^{\prime}\right\}$. Choose admissible multidegrees $w_{0}$ and $\left(w_{v}\right)_{v}$ such that $D_{w_{0}}=$ $D_{w_{v}}=2 v$ and $D_{w_{v^{\prime}}}=\widetilde{P}+v^{\prime}$. Straightforward calculation shows that $D_{v}=D_{w_{0}, w_{v}}^{v}=0$, and $D_{v^{\prime}}=D_{w_{0}, w_{v^{\prime}}}^{v^{\prime}}=Q^{\prime}$. It follows that $b_{v, v^{\prime}}=1, f_{0}, f_{1} \in H^{0}\left(Z_{v}, \mathscr{O}_{Z_{v}}\left(\mathcal{D}_{v}+D_{v}\right)\right)$, and $f_{0}^{\prime}, f_{1}^{\prime} \in H^{0}\left(Z_{v^{\prime}}, \mathscr{O}_{Z_{v^{\prime}}}\left(\mathcal{D}_{v^{\prime}}+D_{v^{\prime}}\right)\right)$. The tuple

$$
\begin{aligned}
\left(\left(\mathscr{O}_{Z_{v}}\left(\mathcal{D}_{v}+D_{v}\right), H_{v}\right),\left(\mathscr{O}_{Z_{v^{\prime}}}\left(\mathcal{D}_{v^{\prime}}+D_{v^{\prime}}\right), H_{v^{\prime}}\right)\right) \\
=\left(\left(\mathscr{O}_{Z_{v}}(2 R), H_{v}\right),\left(\mathscr{O}_{Z_{v^{\prime}}}\left(Q^{\prime}\right), H_{v^{\prime}}\right)\right.
\end{aligned}
$$

is a pre-limit linear series on $\left(X_{0}, \boldsymbol{n}\right)$ with respect to $\left(w_{v}, w_{v^{\prime}}\right)$ (by the calculation below) that maps to $(\mathcal{D}, \mathcal{H})$ under $\mathfrak{F}$. Hence, $(\mathcal{D}, \mathcal{H})$ is a limit linear series on $\mathfrak{C}_{X_{0}, n}$. 
Now we calculate the twisting divisors:

$$
\begin{aligned}
D_{i}^{e, v} & =0, P+Q, P+2 Q, 2 P+3 Q, \ldots, \\
D_{i}^{e, v^{\prime}} & =0, Q^{\prime}, P^{\prime}+2 Q^{\prime}, P^{\prime}+3 Q^{\prime}, \ldots
\end{aligned}
$$

We have $\left.\operatorname{div}^{0}\left(f_{0}\right)\right|_{\{P, Q\}}=P,\left.\operatorname{div}^{0}\left(f_{1}\right)\right|_{\{P, Q\}}=P+Q,\left.\operatorname{div}^{0}\left(f_{0}^{\prime}\right)\right|_{\left\{P^{\prime}, Q^{\prime}\right\}}=0$, and $\left.\operatorname{div}^{0}\left(f_{1}^{\prime}\right)\right|_{\left\{P^{\prime}, Q^{\prime}\right\}}=Q^{\prime}$, which satisfies the condition for pre-limit linear series. It is easy to check that the weak glueing condition is not satisfied for $j=0$, since $\left.\operatorname{div}^{0}\left(f_{0}\right)\right|_{\{P, Q\}}-$ $D_{0}^{e, v}=\left.P \operatorname{but}_{\operatorname{div}^{0}}\left(f_{1}^{\prime}\right)\right|_{\left\{P^{\prime}, Q^{\prime}\right\}}-D_{1}^{e, v^{\prime}}=0$. Thus $(\mathcal{D}, \mathcal{H})$ is not smoothable.

Note that the chain structure $n$ and $X_{0}$ in the above example satisfies the conditions in Theorem 4.3. One can also construct non-smoothable limit linear series on $\mathfrak{C}_{X_{0}, n}$ with $\boldsymbol{n}\left(e_{1}\right)=\boldsymbol{n}\left(e_{2}\right)=1$.

\section{Lifting Divisors on Metric Graphs}

Let $X^{\prime} \rightarrow \operatorname{Spec}(R)$ be a regular smoothing family with generic fiber $X$. Let $G^{\prime}$ be the dual graph with associated metric graph $\Gamma^{\prime}$ where each edge is assigned length 1 . M. Baker [Bak08] constructed a specialization map $\tau: \operatorname{Div}\left(X_{\bar{K}}\right) \rightarrow \operatorname{Div}_{\mathbb{Q}}\left(\Gamma^{\prime}\right)$, where $\bar{K}$ denotes the algebraic closure of $K$ and $\operatorname{Div}_{\mathbb{Q}}\left(\Gamma^{\prime}\right)$ is the set of rational divisors on $\Gamma^{\prime}$, with the property that $r(D) \leq r(\tau(D))$ for all $D \in \operatorname{Div}\left(X_{\bar{K}}\right)$. The question of whether a divisor $D \in \operatorname{Div}_{\mathbb{Q}}\left(\Gamma^{\prime}\right)$ of rank $r(D)$ lifts to a divisor in $X_{\bar{K}}$ of the same rank is not completely solved. A survey of partial results can be found in [BJ16]. The case when $\Gamma^{\prime}$ is a chain of loops with generic edge length is proved liftable in [CJP15] by a thorough examination of divisors on $\Gamma^{\prime}$. In this section we provide a different approach to the lifting problem via the smoothing properties of (pre-)limit linear series on curves with chain structure, for an enlarged scale of $\Gamma^{\prime}$ and certain divisors on $\Gamma^{\prime}$. Note that we do not require $\kappa$ to be algebraically closed, and both Definitions 3.2 and 3.3 remain valid as well as the conclusions we use in this section (Lemma 4.9 and Theorems 4.1 and 4.3).

Let $X_{0}, G, \boldsymbol{n}, w_{0},\left(w_{v}\right)_{v}, \Gamma, \widetilde{X}_{0}$ and $\widetilde{G}$ be as in Notation 3.1. Let $\left(w_{v}^{\text {red }}\right)_{v}$ be as in Theorem 3.9.

Theorem 5.1 Let $X$ be a smooth curve of genus $g$ over K. Suppose there is a regular smoothing family $X^{\prime} \rightarrow \operatorname{Spec}(R)$ with generic fiber $X$ and special fiber $\widetilde{X}_{0}$. Suppose further that $\widetilde{X}_{0}$ only has rational components, and $\left(X_{0}, n\right)$ is as in Theorem 4.3 with $d^{\prime}=\min \{2 g-2, g+1\}$ and that $\bar{G}$ is a chain. Then every rational divisor class $D$ on $\Gamma$ such that $r(D) \leq 1$ lifts to a divisor class of the same rank on $X_{\bar{K}}$.

Proof After a finite base field exchange we can assume that $D$ is a integral and edgereduced divisor of multidegree $w_{0}$. We show that $D$ can be lifted to a pre-limit linear series on $\left(X_{0}, n\right)$ with respect to $\left(w_{v}^{\text {red }}\right)_{v}$, which lifts to a limit linear series with respect to the induced enriched structure on $\widetilde{X}_{0}$. This limit linear series is smoothable, and the corresponding $\mathfrak{g}_{d}^{r}$ on $X$ has underlying divisor specializing to $D$ (up to linear equivalence). 
Let $v_{0}, v_{1}, \ldots, v_{m}$ be the vertices of $G$ as in the following graph, and let $e_{i}$ be the edge of $\bar{G}$ that connects $v_{i-1}$ and $v_{i}$. Let $e_{i}^{1}, \ldots, e_{i}^{a_{i}}$ be the edges in $G$ lying over $e_{i}$ where $1 \leq a_{i} \leq 3$. We can assume for simplicity that $w_{0}=w_{v_{0}}^{\text {red }}$, and hence it is reasonable to denote $w_{i}=w_{v_{i}}^{\text {red }}$.

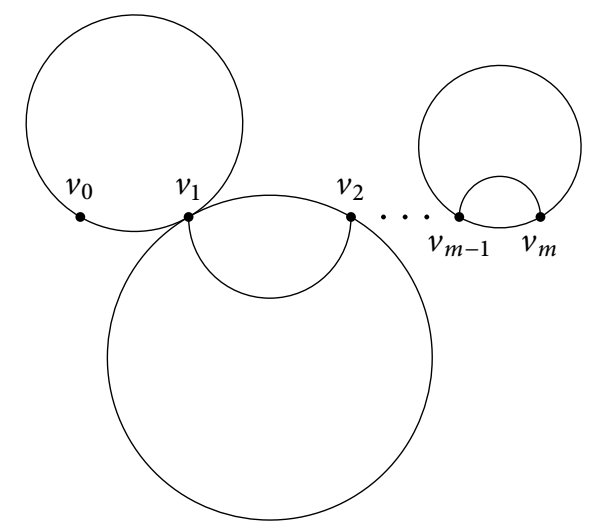

Note that $b_{v_{i-1}, v_{i}}$ is the maximal number $n$ such that the admissible multidegree obtained from $w_{i-1}$ (resp., $\left.w_{i}\right)$ by twisting $n$ times at $\left(e_{i}, v_{i-1}\right)$ (resp., $\left.\left(e_{i}, v_{i}\right)\right)$ is nonnegative at all $v \in V(G)$. Let $Z_{0}, \ldots, Z_{m}$ be the (rational) components of $X_{0}$ corresponding to $v_{0}, \ldots, v_{m}$. Let $d_{i}=\operatorname{deg}\left(D_{w_{i}} \mid v_{i}\right)$. Since $\tau$ is surjective ([Bak08, §2.3]) we can assume that $r(D)=1$. Denote the the pre-limit linear series that we want to construct by $\left(\mathscr{L}_{i}, V_{i}\right)_{i}=\left(\mathscr{O}_{Z_{i}}\left(\mathcal{D}_{i}\right), \operatorname{span}\left(f_{i}^{0}, f_{i}^{1}\right)\right)$.

First take $\mathcal{D}_{0}=P_{0}^{1}+\cdots+P_{0}^{d_{0}}$ with $P_{0}^{j} \in Z_{0} \backslash \mathcal{A}_{e_{1}}^{v_{0}}$. If $b_{v_{0}, v_{1}}>0$, let $f_{0}^{0}=1$ and take $f_{0}^{1}$ such that $\operatorname{ord}_{P_{e_{1}^{v_{0}}}^{j}}\left(f_{0}^{1}\right)=\operatorname{ord}_{P_{e_{1}^{j}}^{v_{0}}}\left(D_{b_{v_{0}, v_{1}}}^{e_{1}, v_{0}}\right)$. If $b_{v_{0}, v_{1}}=0$, let $f_{0}^{0}=1$ and take $f_{0}^{1}$ such that $f_{0}^{1}$ has no zeros or poles at $\left\{P_{e_{1}^{j}}^{v_{0}}\right\}_{j}$ and that $\Pi_{j \neq k}\left(f_{0}^{1}\left(P_{e_{1}^{j}}^{v_{0}}\right)-f_{0}^{1}\left(P_{e_{1}^{k}}^{v_{0}}\right)\right) \neq 0$. Next assume that $\left(\mathscr{L}_{i-1}, V_{i-1}\right)$ is given. Set $f_{i}^{0}=1$.

If $b_{v_{i-1}, v_{i}}=b_{v_{i}, v_{i+1}}=0$ we take $\mathcal{D}_{i}=P_{i}^{1}+\cdots+P_{i}^{d_{i}}$ with $P_{i}^{j} \in Z_{i} \backslash\left(\mathcal{A}_{e_{i}}^{v_{i}} \cup \mathcal{A}_{e_{i+1}}^{v_{i}}\right)$ general. Take $f_{i}^{1}$ such that $f_{i}^{1}$ has no zeros or poles at $\mathcal{A}_{e_{i}}^{v_{i}} \cup \mathcal{A}_{e_{i+1}}^{v_{i}}$ and that

$$
\Pi_{j \neq k}\left(f_{i}^{1}\left(P_{e_{i}^{j}}^{v_{i}}\right)-f_{i}^{1}\left(P_{e_{i}^{k}}^{v_{i}}\right)\right) \cdot \Pi_{j \neq k}\left(f_{i}^{1}\left(P_{e_{i+1}^{j}}^{v_{i}}\right)-f_{i}^{1}\left(P_{e_{i+1}^{k}}^{v_{i}}\right)\right) \neq 0 .
$$

If $b_{v_{i}, v_{i+1}}=0$ and $b_{v_{i-1}, v_{i}}>0$, we take $D_{i}$ as above. Take $f_{i}^{1}$ such that $f_{i}^{1}$ has no zeros or poles at $\mathcal{A}_{e_{i+1}}^{v_{i}}$ and that

$$
\Pi_{j \neq k}\left(f_{i}^{1}\left(P_{e_{i+1}^{j}}^{v_{i}}\right)-f_{i}^{1}\left(P_{e_{i+1}^{k}}^{v_{i}}\right)\right) \neq 0
$$

and that

$$
\operatorname{ord}_{P_{e_{i}^{v_{i}}}^{v_{i}}}\left(f_{i}^{1}\right)=\operatorname{ord}_{P_{e_{i}^{v_{i}}}^{v_{i}}}\left(D_{b_{v_{i-1}, v_{i}}}^{e_{i}, v_{i}}\right) \text {. }
$$

If $b_{v_{i}, v_{i+1}}>0$ and $b_{v_{i-1}, v_{i}}=0$, we take $D_{i}$ as above. Take and $f_{i}^{1}$ such that $f_{i}^{1}$ has no zeros or poles at $\mathcal{A}_{e_{i}}^{v_{i}}$ and that $\Pi_{j \neq k}\left(f_{i}^{1}\left(P_{e_{i}^{j}}^{v_{i}}\right)-f_{i}^{1}\left(P_{e_{i}^{k}}^{v_{i}}\right)\right) \neq 0$ and that $\operatorname{ord}_{P_{e_{i+1}^{j}}^{v_{j}}}\left(f_{i}^{1}\right)=$ $\operatorname{ord}_{P_{e_{i+1}}^{v_{i}}}\left(D_{b_{v_{i}, v_{i+1}}^{e_{i+1}, v_{i}}}^{e_{i}}\right)$. 


$$
\begin{aligned}
& \text { If } b_{v_{i}, v_{i+1}}>0 \text { and } b_{v_{i-1}, v_{i}}>0 \text {, we take } \\
& \qquad \mathcal{D}_{i}=\sum_{j} \operatorname{ord}_{P_{e_{i}^{j}}^{v_{i}}}\left(D_{b_{v_{i-1}, v_{i}}}^{e_{i}, v_{i}}\right) P_{e_{i}^{j}}^{v_{i}}+\mathcal{D}^{\prime}=D_{b_{v_{i-1}, v_{i}}^{e_{i}, v_{i}}}^{v_{i}}+\mathcal{D}^{\prime}
\end{aligned}
$$

with $\mathcal{D}^{\prime}$ an effective divisor supported on $Z_{i} \backslash\left(\mathcal{A}_{e_{i}}^{v_{i}} \cup \mathcal{A}_{e_{i+1}}^{v_{i}}\right)$ such that $\operatorname{deg} \mathcal{D}_{i}=d_{i}$. Take $f_{i}^{1}$ such that

$$
\operatorname{ord}_{P_{i}^{v_{i}}}\left(f_{i}^{1}\right)=-\operatorname{ord}_{e_{i}^{v_{i}}}\left(D_{e_{i}^{j}}^{e_{i}, v_{i}}\right) \quad \text { and } \quad \operatorname{ord}_{P_{v_{i-1}, v_{i}}^{v_{i}}}\left(f_{i}^{1}\right)=\operatorname{ord}_{e_{i+1}^{v_{i}}}\left(D_{e_{i+1}^{j}}^{e_{b_{v_{i}}, v_{i+1}}^{e_{i+1}, v_{i}}}\right) .
$$

Now let $1 \leq i \leq g$. If $b_{v_{i-1}, v_{i}}>0$, then $\operatorname{ord}_{D_{i}^{e_{i}, v_{i-1}}} f_{i-1}^{0}=\operatorname{deg} D_{0}^{e_{i}, v_{i-1}}$ and $\operatorname{ord}_{D_{i}^{e_{i}, v_{i-1}}} f_{i-1}^{1}=$

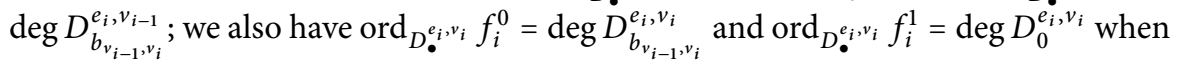
$b_{v_{i}, v_{i+1}}>0$, while $\operatorname{ord}_{D_{i}^{e_{i}, v_{i}}} f_{i}^{1}=\operatorname{deg} D_{b_{v_{i-1}, v_{i}}^{e_{i}, v_{i}}}$ and $\operatorname{ord}_{D_{i}^{e_{i}, v_{i}}} f_{i}^{0}=\operatorname{deg} D_{0}^{e_{i}, v_{i}}$ when $b_{v_{i}, v_{i+1}}=$ 0 . Note that 0 and $b_{v_{i-1}, v_{i}}$ are critical for both $D_{\bullet}^{e_{i}, v_{i-1}}$ and $D_{\bullet}^{e_{i}, v_{i}}$, hence $\left(\mathscr{L}_{i}, V_{i}\right)_{i}$ is a pre-limit linear series with respect to $\left(w_{i}\right)_{v_{i}}$. Moreover, one checks easily that $\left(\mathscr{L}_{i}, V_{i}\right)_{v_{i}}$ satisfies the weak glueing condition.

Now since $r(D)=1$, the Riemann-Roch theorem for graphs [GK08, Proposition 3.1] shows that $\operatorname{deg} D \leq g+1$. If $\operatorname{deg} D \leq 2 g-2$, Lemma 4.9 implies that $\left(\mathscr{L}_{i}, V_{i}\right)_{v_{i}}$ lifts to a limit $\mathfrak{g}_{d}^{1}$ on $\left(X_{0}, \boldsymbol{n}\right)$, which is smoothable by Theorems 4.1 and 4.3. Thus $D$ can be lifted to a rank-one divisor on $X$. If $\operatorname{deg} D>2 g-2$ we can take a divisor $\mathcal{D} \in \operatorname{Div}(X)$ that specializes to $D$ and the Riemann-Roch theorems (on curves and graphs) would imply that $r(\mathcal{D})=r(D)$.

Remark 5.2 If we set $d^{\prime}=2 g-2$ in Theorem 4.3, then as described in [Oss16, Remark 5.4], this recovers precisely the general curves considered in [CJP15]. Thus the Theorem above provides an alternative proof for [CJP15, Theorem 1.1] for divisors of rank less than or equal to one (over a complete discrete valued field). Later we will give another proof of [CJP15, Theorem 1.1] for vertex-avoiding divisors.

Combining this with the tropical Riemann-Roch theorem, we can prove Theorem 5.1 without requiring $r(D) \leq 1$ for $X$ with small genus.

Corollary 5.3 In Theorem 5.1, suppose further that $g \leq 5$. Then any rational divisor class $D$ on $\Gamma$ can be lifted to a divisor class on $X_{\bar{K}}$ of the same rank as $D$.

Proof We can assume that $r(D) \geq 2$ and, by the Riemann-Roch theorem, that $r\left(K_{\Gamma}-D\right) \geq 2$. We have $r\left(K_{\Gamma}\right)=g-1 \geq r(D)+r\left(K_{\Gamma}-D\right) \geq 4$, hence it remains to consider the case $g=5$ and $r(D)=r\left(K_{\Gamma}-D\right)=2$. Now the tropical Clifford theorem [Fac10, Theorem 1] shows that $\operatorname{deg} D=4$ and $D=2 D^{\prime}$ where $r\left(D^{\prime}\right)=1$. It follows from Theorem 5.1 that $D^{\prime}$ is liftable to a divisor of the same rank, hence so is $D$.

We next show the lifting of vertex-avoiding divisors on a generic chain of loops. Since only one proposition is needed in the proof, we refer to [CJP15] for the definition of vertex-avoiding divisors. The idea for the proof came from Sam Payne at a workshop. 
Theorem 5.4 In Theorem 5.1 let $d^{\prime}=2 g-2$ instead. Suppose further that every pair of adjacent vertices is connected by at most two edges. Then every rational vertex-avoiding divisor $D$ on $\Gamma$ lifts to a divisor class on $X_{\bar{K}}$ of the same rank as $D$.

Proof We use the same strategy as in the proof of Theorem 5.1. Suppose $D$ is $v_{0}$ reduced. Let $v_{i}, e_{i}, e_{i}^{j}, w_{i}$ and $d_{i}$ be as in Theorem 5.1. According to [CJP15, Proposition 2.4], for each $0 \leq j \leq r$ there exists a unique divisor $D_{j}$ linearly equivalent to $D$ such that $D_{j}-j v_{0}-(r-j) v_{m}$ is effective, which is obtained by taking a pile of $d_{0}-j$ chips from $v_{0}$ and moving to the right (see the proof of [JP14, Proposition 6.3]), where $d_{0}$ is the coefficient of $D$ at $v_{0}$ as in Theorem 5.1.

Take rational functions $f_{\Gamma}^{j}$ on $\Gamma$ such that $D_{j}=D+\operatorname{div}\left(f_{\Gamma}^{j}\right)$. For $0 \leq i \leq m$ and $0 \leq j \leq r$ let

$$
D_{i}^{j}=\sum_{k} \operatorname{slp}_{e_{i}^{k}, v_{i}}\left(f_{\Gamma}^{j}\right) P_{e_{i}^{k}}^{v_{i}} \quad \text { and } \quad E_{i}^{j}=-\sum_{k} \operatorname{slp}_{e_{i+1}^{k}, v_{i}}\left(f_{\Gamma}^{j}\right) P_{e_{i+1}^{k}}^{v_{i}}
$$

be divisors on $Z_{i}$. Hence $D_{i}^{j}$ represents the chips we get when we try to move the pile of $r$-j chips of $v_{0}$ from $v_{i-1}$ to $v_{i}$, while $E_{i}^{j}$ represents the chips we lost when move the chips from $v_{i}$ to $v_{i+1}$ (note that the size of the pile of chips may change as it moves).

For each $i$, by construction we have

$$
D_{i}^{j}=D_{b_{v_{i-1}, v_{i}}^{e_{i}, v_{i}}}^{e^{\prime}}-D_{m_{i}^{j}}^{e_{i}, v_{i}}
$$

for some $m_{i}^{j}$ critical and $E_{i}^{j}=D_{n_{i}^{j}}^{e_{i+1}, v_{i}}$ for some $n_{i}^{j}$ critical such that $n_{i}^{j}+m_{i+1}^{j}=b_{v_{i}, v_{i+1}}$. The uniqueness of $D_{j}$ shows that the $D_{i}^{j}$ s are distinct for fixed $i$ and so are the $E_{i}^{j}$ s. Now we take $\mathcal{D}_{i}=D_{b_{v_{i-1}, v_{i}}^{e_{i}, v_{i}}}+\mathcal{D}_{i}^{\prime}$ to be a divisor of degree $d_{i}$ on $Z_{v_{i}}$ where $\mathcal{D}_{i}^{\prime} \subset Z_{v_{i}} \backslash \mathcal{A}_{v_{i}}$ is effective and $f_{i}^{j} \in \mathscr{O}_{Z_{v_{i}}}\left(\mathcal{D}_{i}\right)$ such that

$$
\operatorname{ord}_{P_{e_{i}^{v_{i}}}^{v_{i}}}\left(f_{i}^{j}\right)=-\operatorname{ord}_{P_{e_{i}^{v_{i}}}^{v_{i}}}\left(D_{i}^{j}\right) \quad \text { and } \quad \operatorname{ord}_{P_{e_{i+1}^{v_{i}}}}\left(f_{i}^{j}\right)=\operatorname{ord}_{P_{e_{i+1}^{v_{i}}}}\left(E_{i}^{j}\right)
$$

for all possible $k$. Note that the existence of $f_{i}^{j}$ follows from the effectiveness of $D_{j}$. It is easy to check that the tuple $\left(\mathscr{L}_{i}, V_{i}\right)_{v_{i}}=\left(\mathscr{O}_{Z_{v_{i}}}\left(\mathcal{D}_{i}\right), \operatorname{span}\left(\left\{f_{i}^{j}\right\}_{j}\right)\right)_{v_{i}}$ is a pre-limit linear series on $X_{0}$ that satisfies the weak glueing condition, hence it lifts to a limit linear series on $\left(X_{0}, \boldsymbol{n}\right)$. As a result, $D$ can be lifted to a divisor of rank $r(D)$ on $X$.

Acknowledgments The author would like to thank Brian Osserman for introducing this problem and for helpful conversations, and to thank Sam Payne for the idea of the proof of Theorem 5.4 .

\section{References}

[AB15] O. Amini and M. Baker, Linear series on metrized complexes of algebraic curves. Math. Ann. 362(2015), no. 1-2, 55-106. http://dx.doi.org/10.1007/s00208-014-1093-8.

[Bak08] M. Baker, Specialization of linear systems from curves to graphs. Algebra Number Theory 2(2008), no. 6, 613-653. http://dx.doi.org/10.2140/ant.2008.2.613.

[BJ16] M. Baker and D. Jensen, Degeneration of linear series from the tropical point of view and applications. In: Nonarchimedean and tropical geometry, Springer, Cham, 2016, pp. 365-433.

[BS13] M. Baker and F. Shokrieh, Chip-firing games, potential theory on graphs, and spanning trees. J. Combin. Theory Ser. A 120(2013), no. 1, 164-182. http://dx.doi.org/10.1016/j.jcta.2012.07.011. 
[CDPR12] F. Cools, J. Draisma, S. Payne, and E. Robeva, A tropical proof of the Brill-Noether theorem. Adv. Math. 230(2012), no. 2, 759-776. http://dx.doi.org/10.1016/j.aim.2012.02.019.

[CJP15] D. Cartwright, D. Jensen, and S. Payne, Lifting divisors on a generic chain of loops. Canad. Math. Bull. 58(2015), no. 2, 250-262. http://dx.doi.org/10.4153/CMB-2014-050-2.

[Con08] B. Conrad, Several approaches to non-archimedean geometry. In: p-adic geometry, Univ. Lectures Ser., 45, American Mathematical Society, Providence, RI, 2008, pp. 9-63. http://dx.doi.org/10.1090/ulect/045/02.

[Est98] E. Esteves, Linear systems and ramification points on reducible nodal curves. Mat. Contemp. 14(1998), 21-35.

[EH86] D. Eisenbud and J. Harris, Limit linear series: basic theory. Invent. Math. 85(1986), no. 2, 337-371. http://dx.doi.org/10.1007/BF01389094.

[EH87] D. Eisenbud and J. Harris, The Kodaira dimension of the moduli space of curves of genus $\geq 23$. Invent. Math. 90(1987), no. 2, 359-387. http://dx.doi.org/10.1007/BF01388710.

[EM02] E. Esteves and N. Medeiros, Limit canonical systems on curves with two components. Invent. Math. 149(2002), no. 2, 267-338. http://dx.doi.org/10.1007/s002220200211.

[Fac10] L. Facchini, On tropical cliffordâĂŹs theorem. Ric. Mat. 59(2010), no. 2, 343-349. http://dx.doi.org/10.1007/s11587-010-0091-8.

[GK08] A. Gathmann and M. Kerber, A Riemann-Roch theorem in tropical geometry. Math. Z. 259(2008), no. 1, 217-230. http://dx.doi.org/10.1007/s00209-007-0222-4.

[Gie82] D. Gieseker, Stable curves and special divisors: Petri's conjecture. Invent. Math. 66(1982), no. 2, 251-275. http://dx.doi.org/10.1007/BF01389394.

[GH80] P. Griffiths and J. Harris, On the variety of special linear systems on a general algebraic curve. Duke Math. J. 47(1980), no. 1, 233-272.

[HM82] J. Harris and D. Mumford, On the Kodaira dimension of the moduli space of curves. Invent. Math. 67(1982), no. 1, 23-86. http://dx.doi.org/10.1007/BF01393371.

[JP14] D. Jensen and S. Payne, Tropical independence I: shapes of divisors and a proof of the Gieseker-Petri theorem. Algebra Number Theory 8(2014), no. 9, 2043-2066. http://dx.doi.org/10.2140/ant.2014.8.2043.

[Luo11] Y. Luo, Rank-determining sets of metric graphs. J. Combin. Theory Ser. A 118(2011), no. 6, 1775-1793. http://dx.doi.org/10.1016/j.jcta.2011.03.002.

[LM14] Y. Luo and M. Manjunath, Smoothing of limit linear series of rank one on saturated metrized complexes of algebraic curves. Canad. J. Math. 70(2018), no. 3, 628-682. http://dx.doi.org/10.4153/CJM-2017-027-2.

[Mai98] L. Mainò, Moduli space of enriched stable curves. Phd thesis, Harvard University, 1998.

[Oss] B. Osserman, Limit linear series for curves not of compact type. Journal für die reine und angewandte Mathematik (Crelle's journal). to appear.

[Oss16] B. Osserman, Dimension counts for limit linear series on curves not of compact type. Math. Z. 284(2016), no. 1-2, 69-93. http://dx.doi.org/10.1007/s00209-016-1646-5.

[Oss17] B. Osserman, Limit linear series and the Amini-Baker construction, 2017. arxiv:1707.03845.

The Einstein Institute of Mathematics, The Hebrew University of Jerusalem, Edmond J. Safra Campus, 9190401 Givat Ram. Jerusalem,

Israel

e-mail: unihe@ucdavis.edu 